\title{
GENERALIZED DERIVATIVES AND APPROXIMATION BY POLYNOMIALS*
}

BY

W. E. SEWELL

1. Introduction. $\dagger$ Let $E$ be a closed limited point set in the complex $z$ plane, let the complement (with respect to the extended plane) $K$ of $E$ be simply connected, and let $C$ denote the boundary of $E$. In the case where $E$ contains interior points we shall denote by $R$ the limited simply connected region whose closure is $\bar{R} \equiv E$ and whose boundary is $C$. Walsh [1] $\ddagger$ has shown that if $R$ is a Jordan region and if the function $f(z)$ is analytic in $R$ and continuous in $\bar{R}$, then $f(z)$ can be uniformly approximated in $\bar{R}$ by a polynomial in $z$. The best degree of convergence of approximating polynomials to the function depends upon the continuity properties both of the boundary of the region and of the function on the boundary. Reciprocally, under certain conditions continuity properties of the function on the boundary are a consequence of the degree of convergence of approximating polynomials. It is the purpose of this paper to investigate the nature of the relation between continuity properties and degree of convergence.

This investigation can be divided into two parts which we shall call Problem $\alpha$ and Problem $\beta$. In Problem $\alpha$ either the continuity properties of the function $f(z)$, analytic in $R$, are given in the closed limited simply connected region $\bar{R}$, bounded by a Jordan curve $C$, and we study the degree of convergence of certain approximating polynomials in $\bar{R}$, or the degree of convergence in $\bar{R}$ is given and we study the continuity properties of the function on the boundary $C$. In Problem $\alpha$ then we study degree of convergence where the function is not analytic in the closed region.

In Problem $\beta$ we study the degree of convergence of approximating polynomials on interior sets. Let $w=\phi(z)$ map the exterior of $E$ conformally on the exterior of the unit circle, $|w|=1$, so that the points at $\infty$ correspond to each other, then the image in the z-plane of the circle $|w|=\rho, \rho>1$, shall be designated by $C_{\rho}$ (as above $C$ will denote the boundary of $E$ ). If the function $f(z)$ is analytic interior to a particular $C_{\rho}$ and continuous in $\bar{C}_{\rho}$, the closed

* Presented to the Society, December 27, 1934; received by the editors February 29, 1936, and, in revised form, July $21,1936$.

$\dagger$ I wish to express my thanks to Professor J. L. Walsh who proposed this problem to me and under whose direction this paper was written as a thesis at Harvard University. I am also indebted to Dr. W. Seidel who, during Professor Walsh's sabbatical leave, gave me many valuable suggestions.

$\ddagger$ The number in brackets refers to the bibliography at the end of this paper. 
limited simply connected region bounded by $C_{\rho}$, the problem here is the relation between the degree of convergence of approximating polynomials on $E$ and continuity properties of the function $f(z)$ on $\bar{C}_{\rho}$.

Problem $\alpha$ for $E$ a segment of the axis of reals has been widely studied.* Here we follow Montel [1] in describing the continuity properties of the function by the order of its generalized derivative. $\dagger$ We also use to advantage the theory of approximation to functions analytic in closed regions.

In studying degree of convergence we need a close evaluation of the maximum and minimum distances from the boundary $C$ to the curve $C_{\rho}$, which we shall call $D\left(C, C_{\rho}\right)$ and $d\left(C, C_{\rho}\right)$, respectively. Chapter $\mathrm{I}$ is devoted to an investigation of these distance functions for various types of boundaries. We find for $C$ a Jordan curve with corners that $D\left(C, C_{\rho}\right) \leqq M_{2}(\rho-1)^{\text {s }}, d\left(C, C_{\rho}\right) \geqq M_{1}(\rho-1)^{t}$, where $0<s \leqq 1,1 \leqq t<2$. For $E$ an arbitrary closed limited point set whose complement is simply connected we show that $d\left(C, C_{\rho}\right) \geqq M_{1}(\rho-1)^{2}$.

Due to the fact that the generalized derivatives are defined by improper integrals evaluated along rectifiable curves, and to the fact that our method demands the absolute convergence of these integrals, Chapter II is taken up with a discussion, principally by examples, of the types of curves along which the integral $\int_{k}^{z}|z-x|-\alpha|d x|, 0<\alpha<1$, converges. We show, for example, that there are rectifiable curves for which this integral diverges.

In Chapter III the various properties of the generalized derivative which we need for application to approximation are investigated. Also for certain curves relations are established between Lipschitz conditions and generalized derivatives.

Theorems of Bernstein [2], Riesz [1], Markoff [1], and Montel [1] on derivatives of polynomials are extended to generalized derivatives for various types of regions in Chapter IV. In considering Jordan curves with corners we obtain a generalization of a theorem of Szegö [2]. We show that if $P_{n}(z)$ is a polynomial of degree $n$ in $z$ and $\left|P_{n}(z)\right| \leqq M$ on $C$, then for $C$ an analytic Jordan curve $\left|P_{n}^{\alpha}(z)\right| \S \leqq M M_{1}(\alpha, C) n^{\alpha}$, for $C$ a curve with corners $\left|P_{n}^{\alpha}(z)\right|$ $\leqq M M_{1}(\alpha, C) n^{\alpha t}, t<2$, and for $C$ the boundary of a limited simply connected region every boundary point of which is accessible (see \$23)

$$
\left|P_{n}^{\alpha}(z)\right| \leqq M M_{1}(\alpha, C) n^{2 \alpha},
$$

where in all cases $\alpha>0$.

* See e.g., Bernstein [2], Jackson [1], Montel [1].

$\dagger$ Liouville [1], Riemann [1]. See also Weyl [1], Levy [1], Hardy and Littlewood [1, 2], Tamarkin [1], Doetsch [1]. For further references see the articles by Hardy and Littlewood, and Tamarkin.

$\ddagger$ For a complete and excellent exposition of these results see Walsh [1].

$\S f^{\alpha}(z)$ denotes the generalized derivative of order $\alpha$ of $f(z)$. 
In Chapter $\mathrm{V}$ we apply the results of the preceding chapters to obtain theorems on approximation by polynomials leading to solutions of various cases of Problem $\alpha$. For example, if $f(z)$ is analytic in $R$, a limited simply connected region every boundary point of which is accessible (see \$23), and continuous in $\bar{R}$, and if for every $n$ there exists a polynomial $P_{n}(z)$ of degree $n$ in $z$ such that $\left|f(z)-P_{n}(z)\right| \leqq M / n^{\alpha}, z$ in $\bar{R}$, then $f(z)$ has a bounded generalized derivative of order $\alpha^{\prime}<\alpha / t$ on $C$, the boundary of $R$, where $2 \geqq t \geqq 1$, depending on the continuity properties of $C$. It should be noted here that the analyticity of $f(z)$ in $R$, its continuity in $\bar{R}$, and the continuity of an ordinary derivative of $f(z)$ on $C$ imply the continuity of this derivative of $f(z)$ in the closed region bounded by $C ;^{*}$ thus it is sufficient in establishing the results of this paper on approximation to assume the continuity merely of the function $f(z)$ in the closed region.

The last chapter is devoted to a study of Problem $\beta$. Here we consider uniformly bounded functions as well as functions continuous in closed regions. As far as the relation between degree of convergence of a sequence $P_{n}(z)$ to $f(z)$ on $E$ and the continuity properties of $f(z)$ on $C_{\rho}$ we prove, for instance, that if $E$ is a closed limited point set whose complement is simply connected, and if for every $n$ there exists a polynomial $P_{n}(z)$ of degree $n$ in $z$ such that $\left|f(z)-P_{n}(z)\right| \leqq M /\left(n^{\alpha+1} \rho^{n}\right), \alpha>0, \rho>1, z$ on $E$, then $f(z)$ has derivatives of all orders $\alpha^{\prime}<\alpha$ on $C_{\rho}$, and show by example that this is the best result possible in a certain sense.

\section{Chapter I}

\section{The LeVel Curves of the Green's function}

2. Definitions. Let $E, K, C$, and $C_{\rho}$ have the same meaning as above. The curve $C_{\rho}$ is a level curve of the Green's function of $K$ with pole at $\infty$ and as $\rho$ approaches 1 the analytic Jordan curve $C_{\rho}$ approaches the boundary $C$ of $E$, however, if $C$ is not a Jordan curve there may be points of $C$ which $C_{\rho}$ does not approach. Let $P$ be a point of $C$ and define $d\left(P, C_{\rho}\right)$ as the greatest lower bound of the distances from $P$ to the points of $C_{\rho}$. Now we define $d\left(C, C_{\rho}\right)$ as the greatest lower bound of $d\left(P, C_{\rho}\right)$ as $P$ traverses $C$, and $D\left(C, C_{\rho}\right)$ as the least upper bound of $d\left(P, C_{\rho}\right)$ as $P$ traverses $C$. In a similar manner we define $d\left(C_{\rho}, C\right)$ and $D\left(C_{\rho}, C\right)$. We will investigate here the nature of the approach of $C_{\rho}$ to $C$ by studying the functions $d$ and $D$. In the case where $C$ is a Jordan curve all of these functions approach 0 as $\rho$ approaches 1 ; for an arbitrary boundary this is not necessarily true. Although $C_{\rho}$ is defined for every $\rho>1$ we are interested in the behavior of $C_{\rho}$ for $\rho$ near 1 , or at least for $\rho$ uniformly bounded from infinity and hence this condition will be assumed in all our inequalities.

${ }^{*}$ For a detailed discussion of this fact see Walsh and Sewell [1]. 
In the above discussion, we have assumed that the set $E$ is limited, we may consider the case, however, where $R$ is an arbitrary simply connected region, containing, for definiteness, the point $z=0$, and denote by $C$ the boundary of $R$. Let $w=\phi(z)$ map conformally the interior of $R$ on the interior of the unit circle, $|w|=1$, so that the origins correspond to each other, and consider the interior level curve $C_{\rho}$ which is the image in the z-plane, under the inverse map, of the circle $|w|=\rho, 0<\rho<1$. We can define the functions $d$ and $D$ precisely as above and in general the results are valid for both interior and exterior level curves. Of course, in the case where $\bar{R}$ contains the point at $\infty$ we can obtain no evaluation for $D\left(C, C_{\rho}\right)$.

3. Smooth curves. Let $C$ be an analytic Jordan curve and let $w=\phi(z)$, whose inverse is $z=\psi(w)$, map the interior of $C$ on the interior of $|w|=1$. Then we know that*

$$
0<N_{1}<\left|\psi^{\prime}(w)\right|<N_{2}<\infty, \text { for }|w| \leqq 1,
$$

and hence by considering the difference quotient we have

$$
\begin{aligned}
& d\left(C, C_{\rho}\right) \geqq M_{1}|1-\rho|, \\
& D\left(C, C_{\rho}\right) \leqq M_{2}|1-\rho|,
\end{aligned}
$$

where $M_{1}$ and $M_{2}$ are constants depending only on $C$. In the above evaluation we have used the analyticity of $C$ only to establish inequality (3.10) on the first derivative of the mapping function in the closed unit circle. As a matter of fact inequality (3.11) and inequality (3.12) are valid in case the mapping function satisfies the inequality

and the inequality

$$
\left|\frac{\psi\left(w_{1}\right)-\psi\left(w_{2}\right)}{w_{1}-w_{2}}\right| \geqq N_{1}>0,
$$

$$
\left|\frac{\psi\left(w_{1}\right)-\psi\left(w_{2}\right)}{w_{1}-w_{2}}\right| \leqq N_{2}<\infty,
$$

respectively, both holding uniformly for $\left|w_{1}\right| \leqq 1,\left|w_{2}\right| \leqq 1$.

DeFINITION 3.1. If inequalities (3.13) and (3.14) are satisfied, we shall say that "C is a curve of Type S."

The geometric properties of a curve of Type $S$ are of interest. Seidel [1] $\dagger$ has shown that the mapping function possesses the property (3.10), and hence satisfies the inequalities (3.13) and (3.14), provided

${ }^{*} f^{\prime}(z)$ denotes the first derivative of $f(z)$.

† See in particular pp. 213-220. See also Carathéodory [1], Chap. VI; Visser [1]; Kellogg [1]; Warschawski [1]. 


$$
|\omega(s+h)-\omega(s)|<M|h|, \quad M>0,
$$

where $\omega$ denotes the angle between the positively directed tangent and the real axis and $s$ the arc length along the curve.

The same evaluations, (3.11) and (3.12), hold for $d\left(C_{\rho}, C\right)$ and $D\left(C_{\rho}, C\right)$. If suitably interpreted our definition and evaluations are valid for exterior level curves. Thus we have the following theorem:

Theorem 3.2. Let $C$ be a curve of Type $S$. Then we have

$$
d\left(C, C_{\rho}\right) \geqq M_{1}|1-\rho|, \quad D\left(C, C_{\rho}\right) \leqq M_{2}|1-\rho|,
$$

where $C_{\rho}$ is an exterior or interior level curve, and $M_{1}$ and $M_{2}$ are constants depending only on $C$.

4. Curves with corners. Let $C$ be a Jordan curve consisting of a finite number of analytic Jordan arcs meeting in corners with exterior openings $\mu_{k} \pi$, $0<\mu_{k} \leqq 2, k=1,2, \cdots, p$; let $w=\phi(z)$, whose inverse is $z=\psi(w)$, denote the exterior mapping function, and consider the exterior level curves. If $P$ is a point of $C$, not a corner, condition (3.10) is satisfied in a neighborhood of $P$ due to the analyticity of the arc containing $P$, and hence we have inequalities corresponding to (3.11) and (3.12) for $P$, however the constants $M_{1}$ and $M_{2}$ are functions of $P$. In order to obtain a uniform evaluation we must investigate the behavior of $C_{\rho}$ near a corner. Let $z_{0}$ be a corner with exterior opening $\mu \pi, 0<\mu \leqq 2$, and consider the quotient

$$
\theta\left(z_{1}, z_{2}\right)=\frac{\left[\phi\left(z_{1}\right)-\phi\left(z_{2}\right)\right]^{t}}{z_{1}-z_{2}},
$$

where $t=\mu$ if $\mu \geqq 1$, and $t=1$ if $\mu<1, z_{1}$ and $z_{2}$ in the closed exterior of $C$. We can show that $\left|\theta\left(z_{1}, z_{2}\right)\right|$ is uniformly bounded by using the fact that in a suffciently small neighborhood of a point not a corner the difference quotient of the mapping function is bounded in modulus due to the analyticity of the arc, and in the neighborhood of a corner the function $\phi(z)$ can be written in the form*

$$
\phi(z)-\phi\left(z_{0}\right)=\left(z-z_{0}\right)^{1 / \mu} \lambda(z),
$$

where $\lambda(z)$ is analytic in a neighborhood of the corner $z_{0}$ lying outside of $C$, is continuous in the closed neighborhood, and $\lambda\left(z_{0}\right) \neq 0$. This gives us an estimate for both $d\left(C, C_{\rho}\right)$ and $D\left(C, C_{\rho}\right)$.

In fact it is not necessary that the arcs be analytic so long as the difference quotient of the mapping function is bounded in modulus on every proper

\footnotetext{
* Osgood and Taylor [1], pp. 282-283. See also Warschawski [2], p. 324.
} 
sub-arc, not containing an end point, of the arc joining two adjacent corners, and on this basis we will formulate the following definitions:

DEFINITION 4.1. Let $C$ be a Jordan curve composed of a finite number of Jordan arcs meeting in corners $z_{1}, z_{2}, \cdots, z_{p}$, of exterior openings $\mu_{1} \pi, \mu_{2} \pi, \cdots, \mu_{p} \pi, 2>\mu_{1} \geqq \mu_{2} \geqq \cdots \geqq \mu_{p}>0$, and let the difference quotient of the mapping function $w=\phi(z)$, which maps the exterior of $C$ on the exterior of $|w|=1$ so that the points at $\infty$ correspond to each other, be bounded in modulus on each smooth sub-arc. Let $t=\mu_{1}$ if $\mu_{1} \geqq 1$, and $t=1$ if $\mu_{1}<1$. Then we shall say that "C is a curve of Type $t$."

Attention should be called to the fact that in Definition 4.1 we have excluded an exterior opening of $2 \pi$, i.e., $\mu_{1}=2$. This is purely for convenience in later application; in fact all of the results of this paragraph are valid for $\mu_{1}=2$.

Definition 4.2. Under the same conditions as in Definition 4.1 let $s=\mu_{p}$ if $\mu_{p} \leqq 1$, and $s=1$ if $\mu_{p}>1$. Then we shall say that "C is a curve of $T y p e s . "$

And now we can state the following theorems:

THEOREM 4.3. Let $C$ be a curve of Type $t$. Then we have

$$
d\left(C, C_{\rho}\right) \geqq M_{1}(\rho-1)^{t}, \quad \rho>1, \quad 1 \leqq t<2,
$$

where $C_{p}$ is an exterior level curve, and $M_{1}$ is a constant depending only on $C$.

TheOREM 4.4. Let $C$ be a curve of Type s. Then we have

$$
D\left(C, C_{\rho}\right) \leqq M_{2}(\rho-1)^{s}, \quad \rho>1, \quad 0<s \leqq 1,
$$

where $C_{\rho}$ is an exterior level curve, and $M_{2}$ is a constant depending only on $C$.

These results hold for interior as well as exterior level curves if the interpretation of $\mu_{1}, \mu_{2}, \cdots, \mu_{p}$ is suitably modified.

It is to be noted here that we have excluded the case where $C$ has an exterior opening of zero. At such points the function $D$ is large, in fact Szegö [2, pp. 57-59] has shown that a Jordan curve can be constructed with a zero opening for which $D\left(C, C_{\rho}\right)$ decreases at any preassigned rate, however slow.

5. More general regions. In general, if

$$
\left|\frac{\psi\left(w_{1}\right)-\psi\left(w_{2}\right)}{\left(w_{1}-w_{2}\right)^{\alpha}}\right|, \quad \alpha>0, \quad\left|w_{1}\right| \geqq 1, \quad\left|w_{2}\right| \geqq 1,
$$

considering the exterior map, is uniformly bounded we have $D\left(C, C_{\rho}\right)$ $\leqq M_{2}(\rho-1)^{\alpha}$. Under a similar hypothesis on the uniform boundedness of (5.10) from zero we have $d\left(C, C_{\rho}\right) \geqq M_{1}(\rho-1)^{\alpha}$. 
Moreover, if the point $z_{0}$ of $C$ is mapped into a point $w_{0}$ of $|w|=1$, and the radius to $w_{0}$ cuts the circle $|w|=\rho$ in the point $w_{1}$, whose image in the $z$ plane is the point $z_{1}$ on $C_{\rho}$, we have

$$
d\left(z_{0}, C_{\rho}\right) \leqq \int_{z_{1}}^{z_{0}}|d z|=\int_{w_{1}}^{w_{0}}\left|\psi^{\prime}(w)\right||d w|
$$

by using the Schwarz inequality* we have

$$
d\left(z_{0}, C_{\rho}\right) \leqq(\rho-1)^{1 / 2}\left(\int_{\rho}^{1}\left|\psi^{\prime}(w)\right|^{2} d r\right)^{1 / 2} .
$$

Consequently for a region with a boundary such that the square of the derivative of the mapping function is integrable absolutely along each radius, and the integral is uniformly bounded for the entire circumference, we have

$$
D\left(C, C_{\rho}\right) \leqq M_{2}(\rho-1)^{1 / 2} .
$$

For the arbitrary simply connected region it is impossible to find a universal function of $\rho-1$, say $m(\rho-1)$, which approaches 0 with $\rho-1$, and such that $D\left(C, C_{\rho}\right) \leqq M_{2} m(\rho-1)$. In fact, given an arbitrary function of $\rho-1$ with the property mentioned, Szegö [2] has constructed a region (actually a Jordan region) for which $D\left(C, C_{\rho}\right)$ decreases at precisely this rate.

6. The function $d\left(C, C_{\rho}\right)$ for an arbitrary simply connected region. We need the following lemma which follows directly from a result of Szegö [3]:

LEMMA 6.1. Let $R$ be a simply connected region in the z-plane containing the point $z=0$. Let $z=\psi(w)$ map $R$ conformally on $|w|<1$ so that the origins correspond to each other. Then we have $\dagger$

$$
\varliminf_{w \rightarrow e^{i \theta}}|\psi(w)-\psi(\alpha)| \geqq \frac{a}{16}(1-\rho)^{2},
$$

where $0<\rho<1,|\alpha|=\rho$, and $\left|\psi^{\prime}(0)\right|=a$.

An immediate consequence of Lemma 6.1 is

THEOREM 6.2. Let $R$ be an arbitrary simply connected region with boundary $C$. Then for the interior level curves we have

$$
d\left(C, C_{\rho}\right) \geqq M_{1}(1-\rho)^{2}, \quad 0<\rho<1,
$$

where $M_{1}$ depends only on $R$.

* For this method see Ahlfors [1], pp. 7-8.

$\dagger$ lim denotes the lower limit and is used here since $\psi\left(e^{i \theta}\right)$ may not be well defined.

$\ddagger$ Professor Ahlfors has called my attention to the fact that this result contains, in a sense, the following theorem of Lindelö [1] (see e.g., Walsh [1, pp. 27-32]): 
For the exterior level curves we have

THEOREM 6.3. Let $E$, with boundary $C$, be an arbitrary closed limited point set whose complement is simply connected. Then for the exterior level curves we have

$$
d\left(C, C_{\rho}\right) \geqq M_{1}(\rho-1)^{2}, \quad \rho>1,
$$

where $M_{1}$ depends only on $E .^{*}$

7. Relation between $\rho$ and the capacity. Let $E$ be an arbitrary closed limited point set in the $z$-plane whose complement $K$ is simply connected. Let $z=\psi(w)$ map $K$ conformally on $|w|>1$ so that the points at $\infty$ correspond to each other. Then

$$
z=\psi(w)=c w+c_{0}+\frac{c_{1}}{w}+\frac{c_{2}}{w^{2}}+\cdots, c \neq 0 ;
$$

by a rotation $c$ can be made real and positive and we will assume this to be the case. This constant $c$ is known as the capacity, or Robin's constant, or the transfinite diameter, or the outer radius of the set $E$ or of the boundary $C$ of $E$. We shall use the term capacity in this paper. If we denote by $r$ the capacity of $C_{\rho}, \rho>1$, we have

$$
r=\rho c, \dagger
$$

a relation which will be found useful in our later work.

THEOREM. Let $R$ be a limited simply connected region in the z-plane containing the origin. Let $\Delta$ be the greatest diameter of $R$, and let $\delta$ be the shortest distance from $z=0$ to the boundary $C$ of $R$. When $R$ is mapped conformally on the interior of $|w|=1$ so that the origins in the two planes.correspond to each other, every point of $R$ at a distance less than $r$ less than $\delta$ from $C$ corresponds to a point $w$ whose distance from $|w|=1$ is less than

$$
s(r)=\frac{2 \log \Delta-2 \log \delta}{2 \log \Delta-\log (\delta r)} .
$$

Let $a$ be an arbitrary point of $C$, draw a circle of radius $r$ about $a$ and consider a particular $C_{\rho}$ which touches this circle. For this $C_{\rho}$ we have $d\left(C, C_{\rho}\right) \leqq r$, but by Theorem 6.2 we know that $d\left(C, C_{\rho}\right) \geqq M_{1}(1-\rho)^{2}$. Thus we have $\rho \leqq r^{1 / 2} / M_{1}$, and since $\rho$ is precisely $s(r)$ we obtain a much better upper bound for $s(r)$.

Also we can show by Lindelö's method and the above result that any Jordan arc in the circle $|z-a| \leqslant r$ subtends an angle at $w=0$ which is in magnitude less than

$$
4 \sin ^{-1}\left[\left(\frac{M_{1}}{2}\right)^{1 / 2} \Delta^{1 / 8} r^{1 / 8}\right] .
$$

* Professor Ahlfors has pointed out to me that this result holds for any set $E$ whose complement $K$ is connected and regular in the sense that $K$ possesses a Green's function with pole at infinity. For a discussion of such sets see Walsh [1], pp. $65 \mathrm{ff}$.

† See e.g., Walsh [1], pp. 74-75. 


\section{Chapter II \\ IMPROPER INTEGRALS ALONG RECTIFIABLE CURVES}

8. Introduction and definition of curves of Type $W$. In this chapter we will investigate the behavior, convergence, and divergence in particular, of some improper integrals evaluated along rectifiable Jordan arcs or curves, especially of the integral

$$
\int_{k}^{z}|z-x|^{-\alpha}|d x|, \quad 0<\alpha<1,
$$

where the path of integration is an arbitrary rectifiable Jordan arc or curve. The following definition serves as a basis for this investigation:

Definition 8.1. A rectifiable Jordan arc or curve $C$ shall be said to be an "arc or curve of Type $W$ " if the integral (8.10), where the path of integration is along $C$ and $k$ is an arbitrary but fixed point on $C$, converges uniformly in $z, z$ on $C$. For $z=k$ we define $\int_{k}^{z}|z-x|-\alpha|d x|=0$, on a curve or arc of $T y p e W$.

9. Properties of curves of Type $W$. Using the above definition we obtain the following properties:

(9.1) If on a rectifiable Jordan arc or curve $C$ the chord and arc are infinitesimals of the same order uniformly, $C$ is of Type $W$.

This follows from the fact that we can replace the curvilinear integral by a rectilinear integral in the neighborhood of the point $z$.

(9.2) Rectifiable Jordan arcs or curves with continuously turning tangents are of Type $W$.

This is an immediate consequence of (9.1). Thus analytic Jordan arcs or curves are of Type $W$.

(9.3) A curve of Type $S$ is of Type W.

This follows from the fact that (8.10) converges uniformly in the case where $C$ is a circle.

(9.4) Curves of Type $t$ and curves of Type s are of Type $W$.

This is an immediate consequence of (9.3), (9.1), and the fact that one side of a triangle is of the same infinitesimal order as the sum of the other two.

(9.5) Not all rectifiable Jordan arcs and curves are of Type W.

The following example illustrates this proposition: 
Let $z=x+i y=(0,0)$ and $k=(1,0)$, and consider the arc formed by the oblique (non-horizontal) sides of the triangles whose vertices are

$$
\left(\frac{1}{n}, 0\right),\left(\frac{1}{n+1}, 0\right), \quad \text { and }\left(\frac{2 n+1}{2 n(n+1)}, \frac{1}{n^{3 / 2}}\right), \quad n=1,2, \cdots .
$$

(9.6) There are curves of Type $W$ whose chord and arc are not infinitesimals of the same order.

An example illustrating this is obtained by replacing $1 / n^{3 / 2}$ in the above example by $\log n / n^{2}$. It is interesting to note here that if in this example we replace $1 / n^{3 / 2}$ by $1 / n^{\beta}$ the integral (8.10) converges for $\alpha<\beta-1$, the curve is rectifiable for $\beta>1$, and the chord and arc are not infinitesimals of the same order for $\beta<2$.

10. Curves and regions of Type $W^{\prime}$. An arc or curve of Type $W^{\prime}$ is defined as follows:

DeFINITION 10.1. Let $C$ be an arc or curve of Type W. Let $z_{1}$ and $z_{2}$ be arbitrary points, $z_{1} \neq z_{2}$, on $C$. Let $z_{3}$ be a point of $C$ distinct from $z_{1}$ and $z_{2}$, and let $z_{2}$ lie between* $z_{1}$ and $z_{3}$. If constants $M_{1}$ and $M_{2}$, independent of $z_{1}, z_{2}$, and $z_{3}$, exist such that

$$
\int_{z_{1}}^{z_{2}}\left|z_{3}-x\right|^{-\beta}|d x| \leqq M_{1}\left|z_{2}-z_{3}\right|^{-\beta+1}+M_{2}, \quad \beta>0,
$$

for $z_{2}$ arbitrarily near $z_{3}$, we shall say that $C$ is an arc or curve of $T y p e W^{\prime}$.

In case $\beta>1$ the constant $M_{2}$ may be taken as zero since the first term of the right-hand side of the expression (10.10) becomes infinite as the point $z_{2}$ approaches $z_{3}$.

$A$ line segment is an arc of Type $W^{\prime}$. Thus it follows that any rectifiable Jordan arc or curve of which the chord and arc are infinitesimals of the same order uniformly is of Type $W^{\prime}$. Consequently curves of Type $S$, Type $t$, and Type s are of Type $W^{\prime}$.

In connection with curves of Type $W^{\prime}$ we shall introduce a definition which will be found useful later.

DeFinition 10.2. Let $R$ be a limited simply connected region in the z-plane. Let $k$ be an arbitrary but fixed point of $\bar{R}$. If $k$ can be joined to an arbitrary boundary point $\zeta$ of $R$ by an arc $\gamma$ of Type $W^{\prime}$ lying in $\bar{R}$, and if there exist constants $M_{1}$ and $M_{2}$ independent of $\zeta$ such that

* Since $C$ is a Jordan arc or curve the concept of "between" is well defined by fixing a direction on $C$; we may use the parametric representation with a preassigned direction on the line segment or the circle. 


$$
\int_{k}^{z}|\zeta-x|^{-\beta}|d x| \leqq M_{1}|z-\zeta|^{-\beta+1}+M_{2}, \quad \beta>0,
$$

for points $z$ on $\gamma$ and arbitrarily near $\zeta$, we shall say that $R$ is a region of Type $W^{\prime}$.

The definition of a region of Type $W$ is obvious and will not be stated explicitly.

The existence of a single point $k$ in a region of Type $W$ or $W^{\prime}$ is sufficient; from this we can show that any two points of $\bar{R}$ can be joined by an arc of Type $W$ or $W^{\prime}$.

Also it is clear that inequality (10.11) need be assumed only for $z$ near the boundary, since by means, for example, of an interior level curve we can separate off a limited region bounded by an analytic Jordan curve.

\section{Chapter III}

\section{GENERALIZED DERIVATIVES}

11. Definition of the generalized derivative. Let $C$ be a rectifiable Jordan arc or curve in the $z$-plane and let $f(z)$ be continuous on $C$. The ordinary derivative, if it exists, of the function $f(z)$ at a point $z_{0}$ of $C$ is defined as follows:

$$
\left.\lim _{z \rightarrow z_{0}} \frac{f(z)-f\left(z_{0}\right)}{z-z_{0}} \equiv \frac{d f}{d z}\right]_{z=z_{0}} \equiv f^{\prime}\left(z_{0}\right),
$$

$z$ approaching $z_{0}$ along $C$. The generalized derivative, if it exists, of $f(z)$ at a point $z_{0}$ of $C$ is defined as follows:

$$
\begin{aligned}
& D_{z}^{0} f\left(z_{0}\right) \equiv f\left(z_{0}\right) ; \\
& D_{z}^{\alpha} f\left(z_{0}\right) \equiv \frac{1}{\Gamma(-\alpha)} \int_{k}^{z_{0}}\left(z_{0}-x\right)^{-\alpha-1} f(x) d x, \quad \alpha<0 ; \\
& \left.D_{z}^{\alpha} f\left(z_{0}\right) \equiv \frac{d^{p}}{d z^{p}} D_{z}^{\alpha-p} f(z)\right]_{z=z_{0}}, \quad 0 \leqq p-1 \leqq \alpha<p,
\end{aligned}
$$

where $p$ is a positive integer. Here the point $k$ is an arbitrary but fixed point on $C$ if $C$ is a curve, and if $C$ is an arc, $k$ is one of the end points. The path of integration is along $C$ in a fixed direction. The function $D_{z}^{\alpha} f(z), \alpha<0$, is a function of $z$ defined on $C$ and the ordinary derivatives of such a function are defined by (11.10).

If $\alpha$ is a positive integer $D_{z}^{\alpha} f(z)$ reduces to an ordinary derivative of $f(z)$, since the exponent of $(z-x)$ in the integrand vanishes. If $\alpha$ is a negative integer, the function $D_{z}^{\alpha} f(z)$ is the integral of a single valued continuous function evaluated along a rectifiable Jordan arc or curve. 
In the case where $\alpha$ is not an integer the function $(z-x)^{-\alpha-1}$ is multiple valued and care must be taken to choose a branch which varies continuously with $z$ varying along $C$. First let us consider the case where $C$ is a rectifiable Jordan arc and $k$ one of its end points. We will cut the plane from $k$ to $\infty$ along a path whose only point in common with $C$ is $k$ and which goes out to $\infty$ along the positive real axis; this cut makes the plane a simply connected region. Let $\beta=-\alpha-1$; by definition

$$
(z-k)^{\beta}=e^{\beta \log (z-k)}=e^{\beta \log |z-k|+i \beta \arg (z-k)},
$$

and the various branches of this function arise in the $\arg (z-k)$. The function $(z-k)^{\beta}$ is a multiple valued function of $z$ defined throughout the $z$-plane except perhaps at the point $z=k$, where it can be defined by continuity as zero or infinity. We will define $\arg (z-k)$ so that its limit as $z$ approaches $\infty$ along the negative real axis is $\pi$. Thus we have in the simply connected region (cut plane) a well defined single valued analytic function of $z$. Now keep $z$ fixed at an arbitrary point on $C, z \neq k$, and define $\arg (z-x)$ for $x$ on $C$, between $k$ and $z$, in such a way that it takes the value $\arg (z-k)$ determined above for $x=k$. If we hold $z$ fixed on $C$ the function $(z-x)^{\beta}$ for $x$ varying along $C$ from $k$ to $z$ is a single valued analytic function of $x$.

Now consider the case where $C$ is a rectifiable Jordan curve and $k$ is an arbitrary but fixed point on $C$. Cut the $z$-plane from $k$ to $\infty$ along a path whose only point in common with $\bar{C}$, the closed limited region bounded by $C$, is the point $k$, and which goes out to $\infty$ along the positive real axis. Due to the fact that $k$ is a branch point we exclude the value $z=k$ from consideration, in fact for a reason which will be apparent later we will exclude $z$ from a certain preassigned two-dimensional neighborhood of $k$, for any fixed $k$. We will define the branch of $(z-k)^{\beta}$ precisely as above. For $(z-x)^{\beta}$, with $z$ fixed on $C$, we will define the branch as above not only for $x$ on $C$ but also for $x$ interior to $C$. It should be noted that $x$ cannot make a loop around $z$ since $z$ is a boundary point and $x$ is restricted to lie on or interior to $C$.

For the most general case let $R$ be a region of Type $W$ or $W^{\prime}$, let $k$ be an arbitrary point of $\bar{R}$, and cut the plane from $k$ to $\infty$ along a path which goes to $\infty$ along the positive real axis. This cut may contain points of $\bar{R}$ other than $k$, in any event we will exclude $z$ from a two-dimensional strip containing the cut, and restrict $z$ to lie on the boundary of $R$. We will restrict $x$ to paths from $k$ to $z$ which contain no points of the cut. Under these restrictions our definition of the branch as defined above is valid.

It is not necessary to restrict $z$ to the boundary of $R$. We can let $z$ be any point of $\bar{R}$, in fact any point of the plane so long as we restrict $x$ to paths from $k$ to $z$ which do not loop around $z$. 
Thus we have in all cases a well defined single valued branch of the function $(z-x)^{\beta}$ which varies continuously with $z$ under the restrictions prescribed above.

For a given rectifiable Jordan curve $C$ the value of the derivative depends upon the choice of $k$, however, the existence of the derivative is independent of this choice. In fact we have

$$
\int_{k}^{s}(z-x)^{\beta} f(x) d x=\int_{k}^{k_{0}}(z-x)^{\beta} f(x) d x+\int_{k_{0}}^{z}(z-x)^{\beta} f(x) d x,
$$

or

$$
\begin{aligned}
\int_{k}^{z}(z-x)^{\beta} f(x) d x=\int_{k}^{z}(z-x)^{\beta} f(x) d x-\int_{k}^{k_{0}}(z-x)^{\beta} f(x) d x, \\
k \neq k_{0} \neq z .
\end{aligned}
$$

We can evaluate the derivative of the second integral directly since it is a proper integral, and thus we see that if a function has a bounded generalized derivative at a point $z$ for a particular $k \neq z$, then it has a bounded generalized derivative of the same order for any $k_{0}$ between $k$ and $z$. The expression (11.13) indicates the type of function which represents the difference in value of the generalized derivative for two distinct choices of $k$.

Let $f(z)$ be analytic in a region $R$ of Type $W$ and continuous in $\bar{R}$. Let $k$ be a fixed point of $\bar{R}$, and let $C_{1}$ and $C_{2}$ be two distinct arcs of Type $W$ joining $k$ to a boundary point $z$ of $R$, and lying entirely in $\bar{R}$. We assume here that $z \neq k$ and that $z$ does not lie on the cut; we also assume that $C_{1}$ and $C_{2}$ have no points in common with the cut except the point $k$, and, for simplicity, that $C_{1}$ and $C_{2}$ intersect only at $k$ and $z$. If we draw a circle $\gamma$ about $z$ of radius $r$ sufficiently small and let $\Delta$ be the region bounded by $C_{1}, C_{2}$, and one or more $\operatorname{arcs}$ of $\gamma$, we have by Cauchy's integral theorem

$$
\int_{\delta}(z-x)^{\beta} f(x) d x=0,
$$

where $\delta$ denotes the boundary of $\Delta$. Since $f(x)$ is continuous and $\beta>-1$ the modulus of this integral over the arc or arcs of $\gamma$ under consideration approaches 0 as $r$ approaches 0 . Also the integrals along $C_{1}$ and $C_{2}$ converge as $r$ approaches 0 since $C_{1}$ and $C_{2}$ are of Type $W$. Thus we have

$$
\int_{C_{1}}(z-x)^{\beta} f(x) d x=\int_{C_{2}}(z-x)^{\beta} f(x) d x, \quad \beta>-1 .
$$

Consequently the value of the integral is independent of the particular path chosen. 
12. Derivatives of lower order. The proof which we give of the following theorem is only slightly different from that of Montel [1] for functions of a real variable, but it is fundamental in the development and there are some changes which should be pointed out:

THEOREM 12.1. Let $C$ be an arc or curve of Type W. Let $f(z)$ be continuous* on $C$ and admit a bounded generalized derivative of order $\alpha>0$ on the set $C^{m}$, i.e., the set $z$ : $z$ on $C,|z-k|>m>0$, where $m$ is a preassigned positive quantity and $k$ is an arbitrary but fixed point on $C$. Then $f(z)$ admits a bounded derivative of any order $\alpha^{\prime}<\alpha$ on $C^{m}$.

Case I. $0<\alpha^{\prime}<\alpha<1$. By definition

$$
D_{z}^{\alpha} f(z)=F^{\prime}(z), \quad F(z)=\frac{1}{\Gamma(1-\alpha)} \int_{k}^{z}(z-x)^{-\alpha} f(x) d x .
$$

Now form

$$
D_{z}^{\alpha^{\prime}-\alpha} F^{\prime}(z)=\frac{1}{\Gamma\left(\alpha-\alpha^{\prime}\right)} \int_{k}^{z}\left(z-x^{\prime}\right)^{\alpha-\alpha^{\prime}-1} F^{\prime}\left(x^{\prime}\right) d x^{\prime} ;
$$

this expression is the derivative of

$$
\begin{aligned}
I & =\frac{1}{\Gamma\left(\alpha-\alpha^{\prime}\right)} \int_{k}^{z} d z^{\prime} \int_{k}^{z^{\prime}}\left(z^{\prime}-x^{\prime}\right)^{\alpha-\alpha^{\prime}-1} F^{\prime}\left(x^{\prime}\right) d x^{\prime} \\
& =\frac{1}{\Gamma\left(\alpha-\alpha^{\prime}\right)} \int_{k}^{z} F^{\prime}\left(x^{\prime}\right) d x^{\prime} \int_{x^{\prime}}^{z}\left(z^{\prime}-x^{\prime}\right)^{\alpha-\alpha^{\prime}-1} d z^{\prime} .
\end{aligned}
$$

We can change the order of integration since the curve is of Type $W$ and we have absolute convergence. Hence

$$
I=\frac{1}{\left(\alpha-\alpha^{\prime}\right) \Gamma\left(\alpha-\alpha^{\prime}\right)} \int_{k}^{z}\left(z-x^{\prime}\right)^{\alpha-\alpha^{\prime} F^{\prime}\left(x^{\prime}\right) d x^{\prime} .}
$$

Integration by parts is permissible and we have

$$
\begin{aligned}
I & =\frac{1}{\Gamma\left(\alpha-\alpha^{\prime}\right)} \int_{k}^{z}\left(z-x^{\prime}\right)^{\alpha-\alpha^{\prime}-1} F\left(x^{\prime}\right) d x^{\prime} \\
& =\frac{1}{\Gamma\left(\alpha-\alpha^{\prime}\right) \Gamma(1-\alpha)} \int_{k}^{z}\left(z-x^{\prime}\right)^{\alpha-\alpha^{\prime}-1} d x^{\prime} \int_{k}^{x^{\prime}}\left(x^{\prime}-x\right)^{-\alpha} f(x) d x .
\end{aligned}
$$

Since $f(x)$ is continuous and $C$ is of Type $W$ we may change the order of integration and thus

\footnotetext{
${ }^{*}$ For more general functions of a real variable see Tamarkin [1], Theorem 6, p. 227.
} 


$$
I=\frac{1}{\Gamma\left(\alpha-\alpha^{\prime}\right) \Gamma(1-\alpha)} \int_{k}^{z} f(x) d x \int_{x}^{z}\left(x^{\prime}-x\right)^{-\alpha}\left(z-x^{\prime}\right)^{\alpha-\alpha^{\prime}-1} d x^{\prime} .
$$

Now make the transformation $x^{\prime}-x=t(z-x)$, then

$$
I=\frac{1}{\Gamma\left(\alpha-\alpha^{\prime}\right) \Gamma(1-\alpha)} \int_{k}^{z}(z-x)^{-\alpha^{\prime}} f(x) d x \int_{0}^{1}(1-t)^{\alpha-\alpha^{\prime}-1} t^{-\alpha} d t .
$$

It is well known that

$$
\text { 10) } J=\int_{0}^{1}(1-t)^{\alpha-\alpha^{\prime}-1} t^{-\alpha} d t=\frac{\Gamma\left(\alpha-\alpha^{\prime}\right) \Gamma(1-\alpha)}{\Gamma\left(1-\alpha^{\prime}\right)}=B\left(1-\alpha, \alpha-\alpha^{\prime}\right) \text {, }
$$

where the integral is evaluated along the axis of reals between $t=0$ and $t=1$. If the arc in question does not intersect the segment $(0,1)$ or intersects it only a finite number of times, we can use the same method as that employed in the preceding paragraph. If there are an infinite number of intersections, we can draw a circle of radius $\frac{1}{2}$ about the point $t=\frac{1}{2}$; it is clear from our previous results that $J$ evaluated over either the upper or lower half of this circle is the same as $J$ evaluated over the segment $(0,1)$. Also the original arc cannot intersect both the segment $(0,1)$ and the semi-circle an infinite number of times except in a neighborhood of 0 and of 1 since it is by hypothesis rectifiable. In these neighborhoods we can take the limit since all three arcs are of Type $W$ and the integrals over all three arcs approach 0 as the lengths of the arcs approach 0 . Thus we have (12.10) in all cases.

Hence

$$
I=\frac{1}{\Gamma\left(1-\alpha^{\prime}\right)} \int_{k}^{z}(z-x)^{-\alpha^{\prime}} f(x) d x,
$$

and we know by hypothesis that $I$ admits a bounded derivative

$$
\frac{d I}{d z}=D_{z}^{\alpha^{\prime}} f(z)=\frac{1}{\Gamma\left(\alpha-\alpha^{\prime}\right)} \int_{k}^{z}\left(z-x^{\prime}\right)^{\alpha-\alpha^{\prime}-1} F^{\prime}\left(x^{\prime}\right) d x^{\prime} .
$$

CASE II. $0 \leqq p-1 \leqq \alpha^{\prime}<\alpha<p$. By definition

$$
D_{z}^{\alpha} f(z)=F^{(p)}(z),{ }^{*}
$$

where

Let

$$
F(z)=\frac{1}{\Gamma(p-\alpha)} \int_{k}^{z}(z-x)^{p-\alpha-1} f(x) d x .
$$

* The expression $f^{(p)}(z)$, where $p$ is a positive integer, denotes the $p$ th derivative of $f(z) ; f^{(0)}(z) \equiv f(z)$. 


$$
\Phi(z)=D_{z}^{\alpha^{\prime}-\alpha} F^{(p)}(z)=\frac{1}{\Gamma\left(\alpha-\alpha^{\prime}\right)} \int_{k}^{z}\left(z-x^{\prime}\right)^{\alpha-\alpha^{\prime}-1} F^{(p)}\left(x^{\prime}\right) d x^{\prime},
$$

then

$$
\begin{aligned}
\int_{k}^{z} \Phi\left(z^{\prime}\right) d z^{\prime} & =\frac{1}{\Gamma\left(\alpha-\alpha^{\prime}\right)} \int_{k}^{z} d z^{\prime} \int_{k}^{z^{\prime}}\left(z^{\prime}-x^{\prime}\right)^{\alpha-\alpha^{\prime}-1} F^{(p)}\left(x^{\prime}\right) d x^{\prime} \\
& =\frac{1}{\Gamma\left(\alpha-\alpha^{\prime}\right)} \int_{k}^{z} F^{(p)}\left(x^{\prime}\right) d x^{\prime} \int_{x^{\prime}}^{z}\left(z^{\prime}-x^{\prime}\right)^{\alpha-\alpha^{\prime}-1} d z^{\prime} \\
& =\frac{1}{\left(\alpha-\alpha^{\prime}\right) \Gamma\left(\alpha-\alpha^{\prime}\right)} \int_{k}^{z}\left(z-x^{\prime}\right)^{\alpha-\alpha^{\prime} F(p)}\left(x^{\prime}\right) d x^{\prime} .
\end{aligned}
$$

Integration by parts (see above) yields

$$
\begin{aligned}
\int_{k}^{z} \Phi\left(z^{\prime}\right) d z^{\prime}= & -\frac{(z-k)^{\alpha-\alpha^{\prime}} F^{(p-1)}(k)}{\Gamma\left(\alpha-\alpha^{\prime}+1\right)} \\
& +\frac{1}{\Gamma\left(\alpha-\alpha^{\prime}\right)} \int_{k}^{z}\left(z-x^{\prime}\right)^{\alpha-\alpha^{\prime}-1} F^{(p-1)}\left(x^{\prime}\right) d x^{\prime}
\end{aligned}
$$

and likewise

$$
\begin{aligned}
\int_{k}^{z} d z \int_{k}^{z} \Phi\left(z^{\prime}\right) d z^{\prime}= & -\frac{(z-k)^{\alpha-\alpha^{\prime}+1} F^{(p-1)}(k)}{\Gamma\left(\alpha-\alpha^{\prime}+2\right)}-\frac{(z-k)^{\alpha-\alpha^{\prime}} F^{(p-2)}(k)}{\Gamma\left(\alpha-\alpha^{\prime}+1\right)} \\
& +\frac{1}{\Gamma\left(\alpha-\alpha^{\prime}\right)} \int_{k}^{z}\left(z-x^{\prime}\right)^{\alpha-\alpha^{\prime}-1} F^{(p-2)}\left(x^{\prime}\right) d x^{\prime} .
\end{aligned}
$$

Finally

$$
\begin{aligned}
\underbrace{\int_{k}^{z} d z \int \cdots \int_{k}^{z}}_{p-1} \Phi\left(z^{\prime}\right) d z^{\prime}=(z-k)^{\alpha-\alpha^{\prime}} Q_{p-1}(z) & \\
& +\frac{1}{\Gamma\left(\alpha-\alpha^{\prime}\right)} \int_{k}^{z}\left(z-x^{\prime}\right)^{\alpha-\alpha^{\prime}-1} F\left(x^{\prime}\right) d x^{\prime}
\end{aligned}
$$

where $Q_{p-1}(z)$ is a polynomial of degree $p-1$ in $z$. Then

$$
\begin{aligned}
I & =\frac{1}{\Gamma\left(\alpha-\alpha^{\prime}\right)} \int_{k}^{z}\left(z-x^{\prime}\right)^{\alpha-\alpha^{\prime}-1} F\left(x^{\prime}\right) d x^{\prime} \\
& =\frac{1}{\Gamma\left(\alpha-\alpha^{\prime}\right) \Gamma(p-\alpha)} \int_{k}^{z}\left(z-x^{\prime}\right)^{\alpha-\alpha^{\prime}-1} d x^{\prime} \int_{k}^{x^{\prime}}\left(x^{\prime}-x\right)^{p-\alpha-1} f(x) d x \\
& =\frac{1}{\Gamma\left(\alpha-\alpha^{\prime}\right) \Gamma(p-\alpha)} \int_{k}^{z} f(x) d x \int_{x}^{z}\left(z-x^{\prime}\right)^{\alpha-\alpha^{\prime}-1}\left(x^{\prime}-x\right)^{p-\alpha-1} d x^{\prime}
\end{aligned}
$$


By making the same change of variable as above we get

and thus

$$
I=\frac{1}{\Gamma\left(p-\alpha^{\prime}\right)} \int_{k}^{z}(z-x)^{p-\alpha-1} f(x) d x,
$$

$$
\frac{d^{p} I}{d z^{p}}=\Phi(z)-\frac{d^{p}}{d z^{p}}\left[(z-k)^{\alpha-\alpha^{\prime}} Q_{p-1}(z)\right]=D_{z}^{\alpha^{\prime}} f(z),
$$

and this is bounded in modulus since $|z-k|>m>0$.

CASE III. $\alpha=p$. For this case put

$$
\Phi(z)=\frac{1}{\Gamma(p-\alpha)} \int_{k}^{z}(z-x)^{p-\alpha^{\prime}-1} f^{(p)}(x) d x,
$$

and

$$
I=\frac{1}{\Gamma\left(p-\alpha^{\prime}\right)} \int_{k}^{z}(z-x)^{p-\alpha^{\prime}-1} f(x) d x, \quad p-1 \leqq \alpha^{\prime}<p .
$$

Then

$$
\frac{d^{p} I}{d z^{p}}=\Phi(z)+\frac{d^{p}}{d z^{p}}\left[(z-k)^{p-\alpha^{\prime}} Q_{p-1}(z)\right]=D_{z}^{\alpha^{\prime}} f(z) .
$$

Thus the proof is complete for $\alpha>0$ and $\alpha-\alpha^{\prime} \leqq 1$. If $\alpha-\alpha^{\prime}>1$, we can take $\alpha^{\prime \prime}=p-1$ and by applying the above result a finite number of times obtain the result for an arbitrary $\alpha^{\prime}<\alpha$.

13. General properties. Using methods similar to the ones above we will prove

THEOREM 13.1. Let $C$ be an arc of Type $W$. Let $f(z)$ be continuous on $C$ and have a bounded derivative of order $\alpha+\beta$ on $C^{m}$. Then we have

$$
D_{z}^{\alpha}\left\{D_{z}^{\beta} f(z)\right\}=D_{z}^{\alpha+\beta} f(z), \beta<0, \text { z on } C^{m} .
$$

For $\alpha=0$ the result is immediate. For $\alpha<0$ we have

$$
\begin{aligned}
D_{z}^{\alpha}\left\{D_{z}^{\beta} f(z)\right\} & =\frac{1}{\Gamma(-\alpha) \Gamma(-\beta)} \int_{k}^{z}\left(z-x^{\prime}\right)^{-\alpha-1} d x^{\prime} \int_{k}^{x^{\prime}}\left(x^{\prime}-x\right)^{-\beta-1} f(x) d x \\
& =\frac{1}{\Gamma(-\alpha) \Gamma(-\beta)} \int_{k}^{z} f(x) d x \int_{x}^{z}\left(x^{\prime}-x\right)^{-\beta-1}\left(z-x^{\prime}\right)^{-\alpha-1} d x^{\prime} \\
& =\frac{1}{\Gamma(-\alpha-\beta)} \int_{k}^{z}(z-x)^{-\alpha-\beta-1} f(x) d x=D_{z}^{\alpha+\beta} f(z) .
\end{aligned}
$$

For $0 \leqq p-1 \leqq \alpha<p$, we have 


$$
D_{z}^{\alpha}\left\{D_{z}^{\beta} f(z)\right\}=\frac{d^{p}}{d z^{p}} D_{z}^{\alpha-p}\left\{D_{z}^{\beta} f(z)\right\}=\frac{d^{p}}{d z^{p}} D_{z}^{\alpha+\beta-p} f(z) .
$$

If $0 \leqq p-1 \leqq \alpha+\beta<p$, the result follows by definition; if this is not the case, the exponent of $(z-x)$ in the integrand is positive and we can remove the additional integers by the ordinary rules of differentiation.

This theorem is proved by Levy [1] for $C$ a line segment and for $\alpha<0$, $\beta<0$.

Our next result is

THEOREM 13.2. Let $C$ be an arc of Type $W$. Let $f(z)$ be continuous on $C$ and have a bounded derivative of order $\alpha, 0<\alpha \leqq 1$, on $C^{m}$. Also let $f(k)=0$. Then we have

$$
D_{z}^{-\alpha}\left\{D_{z}^{\alpha} f(z)\right\}=f(z), \quad z \text { on } C^{m} .
$$

For $\alpha=1$ the result is immediate. For $\alpha<1$ we have by definition

$$
D_{z}^{\alpha} f(z)=\frac{d}{d z} D_{z}^{\alpha-1} f(z)=\frac{d}{d z} \frac{1}{\Gamma(1-\alpha)} \int_{k}^{z}(z-x)^{-\alpha} f(x) d x .
$$

Let

$$
F(z)=\frac{1}{\Gamma(1-\alpha)} \int_{k}^{z}(z-x)^{-\alpha} f(x) d x .
$$

By hypothesis $F^{\prime}(z)$ is bounded. Let

$$
\Phi(z)=D_{z}^{-\alpha} F^{\prime}(z)=\frac{1}{\Gamma(\alpha)} \int_{k}^{z}\left(z-x^{\prime}\right)^{\alpha-1} F^{\prime}\left(x^{\prime}\right) d x^{\prime} .
$$

The function $\Phi(z)$ is the derivative of

$$
\begin{aligned}
I & =\frac{1}{\Gamma(\alpha)} \int_{k}^{z} d z \int_{k}^{z}\left(z-x^{\prime}\right)^{\alpha-1} F^{\prime}\left(x^{\prime}\right) d x^{\prime} \\
& =\frac{1}{\Gamma(\alpha)} \int_{k}^{z} F^{\prime}\left(x^{\prime}\right) d x^{\prime} \int_{x^{\prime}}^{z}\left(z-x^{\prime}\right)^{\alpha-1} d z \\
& =\frac{1}{\alpha \Gamma(\alpha)} \int_{k}^{z}\left(z-x^{\prime}\right)^{\alpha} F^{\prime}\left(x^{\prime}\right) d x^{\prime},
\end{aligned}
$$

and integration by parts yields

$$
I=\left.\left(z-x^{\prime}\right)^{\alpha} F\left(x^{\prime}\right)\right|_{k} ^{z}+\frac{1}{\Gamma(\alpha)} \int_{k}^{z}\left(z-x^{\prime}\right)^{\alpha-1} F\left(x^{\prime}\right) d x^{\prime} .
$$

We have $F(k)=0$ as defined by $\lim _{z \rightarrow k} F(z)$. Hence 


$$
\begin{aligned}
I & =\frac{1}{\Gamma(\alpha)} \int_{k}^{z}\left(z-x^{\prime}\right)^{\alpha-1} F\left(x^{\prime}\right) d x^{\prime} \\
& =\frac{1}{\Gamma(\alpha) \Gamma(1-\alpha)} \int_{k}^{z}\left(z-x^{\prime}\right)^{\alpha-1} d x^{\prime} \int_{k}^{x^{\prime}}\left(x^{\prime}-x\right)^{-\alpha} f(x) d x \\
& =\frac{1}{\Gamma(\alpha) \Gamma(1-\alpha)} \int_{k}^{z} f(x) d x \int_{z}^{z}\left(z-x^{\prime}\right)^{\alpha-1}\left(x^{\prime}-x\right)^{-\alpha} d x^{\prime}=\int_{k}^{z} f(x) d x .
\end{aligned}
$$

Thus

$$
D_{z}^{-\alpha} F^{\prime}(z)=D_{z}^{-\alpha}\left\{D_{z}^{\alpha} f(z)\right\}=\Phi(z)=\frac{d I}{d z}=\frac{d}{d z} \int_{k}^{z} f(x) d x=f(z) .
$$

For $\alpha>1$ additional conditions on the original function $f(z)$ are involved. The same method can be used to investigate this case. If $f(k)=f^{\prime}(k)=\ldots$ $=f^{(n)}(k)=0$, and if $f(z)$ has bounded ordinary derivatives of sufficiently high order Theorem 13.2 can be extended to values of $\alpha \leqq n$. The case where $0<\alpha \leqq 1$ is the most interesting, consequently the details of the extension will not be included here.

As an immediate consequence of the definition we have

THEOREM 13.3. Let the functions $f_{1}(z)$ and $f_{2}(z)$ be continuous and have bounded derivatives of order $\alpha>0$ on $C^{m}$, where $C$ is an arc of Type $W$. Then we have

$$
D_{z}^{\alpha}\left\{f_{1}(z)+f_{2}(z)\right\}=D_{z}^{\alpha} f_{1}(z)+D_{z}^{\alpha} f_{2}(z) .
$$

\section{We have also}

TheоReм 13.4. Let $C$ be an arc of Type $W$. Let $f(z)$ be continuous on $C$ and have a bounded derivative of order $\alpha, 0 \leqq p-1 \leqq \alpha<p$, on $C^{m}$. Then $f^{(p-1)}(z)$ has a bounded derivative of order $\alpha-p+1$ on $C^{m}$.

By Theorem 12.1 we know that $f^{(p-1)}(z)$ exists and is bounded and by definition

$$
\begin{aligned}
D_{z}^{\alpha-p+1} f^{(p-1)}(z) & =\frac{d}{d z} D_{z-p}^{\alpha-p-1)}(z) \\
& =\frac{d}{d z}\left\{\frac{1}{\Gamma(p-\alpha)} \int_{k}^{z}(z-x)^{p-\alpha-1} f^{(p-1)}(x) d x\right\} .
\end{aligned}
$$

We have to show that

$$
\Phi(z)=\frac{1}{\Gamma(p-\alpha)} \int_{k}^{z}(z-x)^{p-\alpha-1} f^{(p-1)}(x) d x
$$


has a bounded derivative, with the hypothesis that $\int_{k}^{z}(z-x)^{p-\alpha-1} f(x) d x$ has a bounded $p$ th derivative. If we can prove that

$$
\underbrace{\int_{k}^{z} d z \int \cdots \int_{k}^{z}}_{p-1} \Phi(z) d z
$$

has a bounded $p$ th derivative, the theorem is established. We have

$$
\begin{aligned}
& \frac{1}{\Gamma(p-\alpha)} \int_{k}^{z} d z \int_{k}^{z}(z-x)^{p-\alpha-1} f^{(p-1)}(x) d x \\
& \quad=\frac{1}{\Gamma(p-\alpha)} \int_{k}^{z} f^{(p-1)}(x) d x \int_{x}^{z}(z-x)^{p-\alpha-1} d z \\
& \quad=\frac{1}{(p-\alpha) \Gamma(p-\alpha)} \int_{k}^{z}(z-x)^{p-\alpha} f^{(p-1)}(x) d x, \\
& =-\frac{(z-k)^{p-\alpha} f^{(p-2)}(k)}{\Gamma(p-\alpha+1)}+\frac{1}{\Gamma(p-\alpha)} \int_{k}^{z}(z-x)^{p-\alpha-1} f^{(p-2)}(x) d x,
\end{aligned}
$$

integrating by parts. Likewise

$$
\begin{aligned}
\int_{k}^{z} d z \int_{k}^{z} \Phi(z) d z= & -\frac{(z-k)^{p-\alpha+1} f^{(p-2)}(k)}{\Gamma(p-\alpha+2)}-\frac{(z-k)^{p-\alpha} f^{(p-3)}(k)}{\Gamma(p-\alpha+1)} \\
& +\frac{1}{\Gamma(p-\alpha)} \int_{k}^{z}(z-x)^{p-\alpha-1} f^{(p-3)}(x) d x
\end{aligned}
$$

Finally

$$
\begin{aligned}
\underbrace{\int_{k}^{z} d z \int \cdots \int_{k}^{z}}_{p-1} \Phi(z) d z= & (z-k)^{p-\alpha} Q_{p-2}(z) \\
& +\frac{1}{\Gamma(p-\alpha)} \int_{k}^{z}(z-x)^{p-\alpha-1} f(x) d x .
\end{aligned}
$$

The function $Q_{p-2}(z)$ is a polynomial of degree $p-2$ in $z$. Since the expression on the right has a bounded $p$ th derivative for $|z-k|>m>0$, the expression on the left does also and the proof is complete.

A related theorem is the following:

Theorem 13.5. Let $C$ be an arc of Type $W$. Let $f^{(p-1)}(z)$ be continuous on $C$ and have a bounded derivative of order $\alpha-p+1,0 \leqq p-1 \leqq \alpha<p$, on $C^{m}$. Then $f(z)$ has a bounded derivative of order $\alpha$ on $C^{m}$.

We know that 


$$
\frac{d}{d z} \int_{k}^{z}(z-x)^{p-\alpha-1} f^{(p-1)}(x) d x
$$

is bounded and we have to prove that

$$
\frac{d^{p}}{d z^{p}} \int_{k}^{z}(z-x)^{p-\alpha-1} f(x) d x
$$

is bounded. Integrating by parts we have

$$
\Phi(z)=\int_{k}^{z}(z-x)^{p-\alpha-1} f(x) d x=\frac{(z-k)^{p-\alpha} f(k)}{p-\alpha}+\int_{k}^{z}(z-x)^{p-\alpha} f^{\prime}(x) d x,
$$

and integrating the second integral on the right by parts yields

$$
\Phi(z)=\frac{(z-k)^{p-\alpha} f(k)}{p-\alpha}+\frac{(z-k)^{p-\alpha+1} f^{\prime}(k)}{p-\alpha+1}+\int_{k}^{z}(z-x)^{p-\alpha+1} f^{(2)}(x) d x .
$$

Continuing this process we arrive at

$$
\begin{aligned}
\Phi(z)= & \frac{(z-k)^{p-\alpha} f(k)}{p-\alpha}+\frac{(z-k)^{p-\alpha+1} f^{\prime}(k)}{p-\alpha+1}+\cdots \\
& +\frac{(z-k)^{2 p-\alpha-2} f^{(p-2)}(k)}{2 p-\alpha-2}+\int_{k}^{z}(z-x)^{2 p-\alpha-2} f^{(p-1)}(x) d x .
\end{aligned}
$$

We know that each of the terms on the right exclusive of the integral has a bounded $p$ th derivative on $C^{m}$ and thus there remains only to show that this integral has a bounded $p$ th derivative. We can differentiate under the integral sign $p-1$ times and the result is

$$
\int_{k}^{z}(z-x)^{p-\alpha-1} f^{(p-1)}(x) d x .
$$

By hypothesis this has a bounded first derivative and the result is established. There are similar theorems for $f^{(p-2)}(z)$, etc.

The proof of the following theorem is similar in method to that used in showing that the Beta function [see (12.10)] can be evaluated along curves of Type $W$ (see also $\$ 11)$ :

THEOREM 13.6. Let $f(z)$ be analytic in a region bounded by a curve $C$ of Type $W$ and continuous in the corresponding closed region except for a finite number of algebraic singularities of orders less than 1. Then for a particular branch of the function we have $\int_{c} f(z) d z=0$.

14. Invariance of order under conformal transformation. Let $C$ be an analytic Jordan curve in the $z$-plane and let $k$ be a fixed point on $C$. Let 
$w=\phi(z)$ map the closed region bounded by $C$ conformally on the closed unit circle in the w-plane. It is well known that $\phi(z)$ is analytic on $C$, and that $z=\psi(w)$, the inverse of $w=\phi(z)$, is analytic on $|w|=1$. Let $f(z)$ be continuous on $C$ and have a bounded derivative of order $\alpha>0$ on $C^{m}$. We will show that $f(\psi(w))=F(w)$ has a bounded derivative of the same order $\alpha$ on the arc of the unit circle, $|w|=1$, corresponding to $C^{m}$ under the conformal map, the path of integration being the image in the w-plane of the path of integration in the z-plane.

CASE I. $0<\alpha<1$. By definition

$$
D_{z}^{\alpha} f(z)=\frac{d}{d z} \frac{1}{\Gamma(1-\alpha)} \int_{k}^{z}(z-x)^{-\alpha} f(x) d x,
$$

and by hypothesis

$$
g(z)=\int_{k}^{z}(z-x)^{-\alpha} f(x) d x
$$

has a bounded first derivative with respect to $z$. Then

$$
\begin{aligned}
g(\psi(w)) & =\int_{l}^{w}[\psi(w)-\psi(\omega)]^{-\alpha} F(\omega) \psi^{\prime}(\omega) d \omega \\
& =\int_{l}^{w}(w-\omega)^{-\alpha}\left[\frac{\psi(w)-\psi(\omega)}{w-\omega}\right]^{-\alpha} F(\omega) \psi^{\prime}(\omega) d \omega
\end{aligned}
$$

has a bounded first derivative with respect to $w$, where $k$ on $C$ is mapped into $l$ on $|w|=1$. The function

$$
P(w, \omega)=\left[\frac{\psi(w)-\psi(\omega)}{w-\omega}\right]^{-\alpha} \psi^{\prime}(\omega)
$$

is an analytic function of $w$ and $\omega$ and is different from 0 . We may write it in the form

$$
\begin{aligned}
P(w, \omega) & =P(w, w)+\frac{P(w, \omega)-P(w, w)}{w-\omega}(w-\omega) \\
& =P(w, w)+Q(w, \omega)(w-\omega),
\end{aligned}
$$

where $P(w, w) \neq 0$ and is defined as the limit of $P(w, \omega)$ as $\omega$ approaches $w$. Thus we have

$$
\begin{aligned}
g(\psi(w)) & =G(w)=\int_{l}^{w}(w-\omega)^{-\alpha}[P(w, w)+Q(w, \omega)(w-\omega)] F(\omega) d \omega \\
& =P(w, w) \int_{l}^{w}(w-\omega)^{-\alpha} F(\omega) d \omega+\int_{l}^{w}(w-\omega)^{-\alpha+1} Q(w, \omega) F(\omega) d \omega .
\end{aligned}
$$


By hypothesis $G(w)$ has a bounded derivative with respect to $w$. Now set

$$
A(w)=\int_{l}^{w}(w-\omega)^{-\alpha+1} Q(w, \omega) F(\omega) d \omega .
$$

By Leibnitz' rule we have

$$
\begin{aligned}
\frac{d A(w)}{d w} & =\int_{l}^{w}(w-\omega)^{-\alpha+1} Q_{w}(w, \omega) F(\omega) d \omega \\
& +\int_{l}^{w}(-\alpha+1)(w-\omega)^{-\alpha} Q(w, \omega) F(\omega) d \omega .
\end{aligned}
$$

The integral on the right converges absolutely and hence the derivative exists. It follows that $P(w, w) \int_{l}^{w}(w-\omega)^{-\alpha} F(\omega) d \omega$ has a bounded derivative with respect to $w$ and, since $P(w, w) \neq 0$ and has a derivative, we have shown that for $0<\alpha<1$ the function $F(w)$ has a bounded derivative of order $\alpha$.

CASE II. $0 \leqq p-1 \leqq \alpha<p$. If $\alpha=p-1$ the result is immediate. If $\alpha>p-1$ we know by Theorem 12.1 that $f^{(p-1)}(z)$ is bounded and it follows that $F^{(p-1)}(w)$ is bounded. In fact we know that $f^{(p-1)}(z)$ has a bounded derivative of order $\alpha-p+1$ by Theorem 13.4. The expression for $F^{(p-1)}(w)$ is a polynomial in $f^{(1)}, f^{(2)}, \cdots, f^{(p-1)}, \psi^{(1)}, \psi^{(2)}, \cdots, \psi^{(p-1)}$, and we can treat each separate integral as in Case I and the proof is complete.

15. Extension of the Cauchy integral formula. We obtain a new expression for the generalized derivative from the following

Theorem 15.1. Let $C$ be an arc of Type $W, k$ a fixed point on $C$, and $z$ an arbitrary point on $C, z \neq k$. Let $f(z)$ be analytic on and within a rectifiable Jordan curve $\gamma$ which passes through $k$ and contains in its interior that portion of $C$ between $k$ and $z$. Then we have

$$
D_{z}^{\alpha} f(z)=\frac{\Gamma(\alpha+1)}{2 \pi i} \int_{\gamma} \frac{f(t) d t}{(t-z)^{\alpha+1}}, \quad \alpha>0,
$$

where the branch of $(t-z)^{\alpha+1}$ is the one chosen in the original definition with $t$ varying along $C$ from $k$ to $z$.

This is a generalization of Cauchy's integral formula for derivatives and the proof follows that of Montel [1] for $C$ a segment of the axis of reals. By virtue of (11.14) for $C$ of Type $W$ we may replace it by $C_{1}$ of Type $W$, and thus it is only necessary that $\gamma$ pass through $k$ and contain in its interior the point $z$ and an arc of Type $W$ joining $k$ to $z$, so long as $f(z)$ is analytic on and within $\gamma$, in fact it is sufficient for $f(z)$ to be analytic interior to $\gamma$ and continuous in the closed limited region bounded by $\gamma$. For future reference we will state the following more general theorem: 
THEOREM 15.2. Let $f(z)$ be analytic in the interior of a rectifiable Jordan curve $\gamma$ which passes through $k$ and contains in its interior the point $z$ and an arc $C$ of $T y p e W$ joining $k$ to $z$, and let $f(z)$ be continuous in the closed limited region bounded by $\gamma$. Then we have

$$
D_{z}^{\alpha} f(z)=\frac{\Gamma(\alpha+1)}{2 \pi i} \int_{\gamma} \frac{f(t) d t}{(t-z)^{\alpha+1}}, \quad \alpha>0,
$$

where the branch of $(t-z)^{\alpha+1}$ is determined on $C$.

16. A theorem on convergence. The following theorem has important application later:

THEOREM 16.1. Let $C$ be an arc or curve of Type W. Let $f_{1}(z), f_{2}(z), \cdots$ be a sequence of uniformly bounded functions which converges uniformly to $f(z)$ on $C$. Let $f_{1}^{\alpha}(z), f_{2}^{\alpha}(z), \cdots, \alpha>0$, converge uniformly on $C^{m}$. Then the sequence $f_{1}^{\alpha}(z), f_{2}^{\alpha}(z), \ldots$ has for a limit $f^{\alpha}(z)$ on $C^{m}$.

Montel [1] proves this theorem for $C$ a segment of the axis of reals and the modifications necessary to establish this more general result are obvious, hence the proof will not be included here.

17. Lipschitz conditions and generalized derivatives. We begin with the following theorem:*

THEOREM 17.1. Let $C$ be an arc or curve of Type $W$. Let $f(z)$ satisfy a Lipschitz condition $\dagger$ of order $\alpha, 1 \geqq \alpha>0$, on $C$. Then $f(z)$ has a bounded derivative of every order $\beta<\alpha$ on $C^{m}$.

For $\beta \leqq 0$ the result is obvious. For $\beta>0$ we have to show that

$$
\int_{k}^{z}(z-x)^{-\beta} f(x) d x
$$

has a bounded derivative. Consider

$$
\begin{aligned}
I(z) & =\int_{k}^{z-\epsilon}(z-x)^{-\beta} f(x) d x . \\
\frac{d I}{d z} & =\epsilon^{-\beta} f(z-\epsilon)-\beta \int_{k}^{z-\epsilon}(z-x)^{-\beta-1} f(x) d x \\
& =\epsilon^{-\beta}[f(z-\epsilon)-f(z)]+\beta \int_{k}^{z-\epsilon}[f(z)-f(x)](z-x)^{-\beta-1} d x .
\end{aligned}
$$
591.

* For the case where $C$ is a straight line the proof is due to Hardy and Littlewood [1], pp. 590-

† The function $f(z)$ satisfies a Lipschitz condition of order $\alpha$ on a set $E$ if for $z_{1}$ and $z_{2}$ arbitrary points on $E$ we have $\left|f\left(z_{1}\right)-f\left(z_{2}\right)\right| \leqq M\left|z_{1}-z_{2}\right|^{\alpha}$, where $M$ is a constant independent of $z_{1}$ and $z_{2}$. 
The first term on the right approaches 0 as $\epsilon$ approaches 0 by the hypothesis of a Lipschitz condition of order $\alpha$ on the function $f(z)$. Furthermore

$$
\begin{aligned}
\left|\int_{k}^{z-\epsilon}[f(z)-f(x)](z-x)^{-\beta-1} d x\right| & \leqq \int_{k}^{z-\epsilon}|f(z)-f(x)||z-x|^{-\beta-1}|d x| \\
& \leqq \int_{k}^{z-\epsilon} M|z-x|^{\alpha}|z-x|^{-\beta-1}|d x| \\
& =M \int_{k}^{z-\epsilon}|z-x|^{\alpha-\beta-1}|d x| .
\end{aligned}
$$

The last integral converges and the proof is complete.

Now we will consider the case where $f^{(p-1)}(z)$ satisfies a Lipschitz condition of order $\alpha-p+1,0 \leqq p-1 \leqq \alpha<p$, on $C$. From above we know that $f^{(p-1)}(z)$ has a bounded derivative of every order $\beta-p+1<\alpha-p+1$, and hence by Theorem 13.5 the function $f(z)$ has a bounded derivative of every order $\beta<\alpha$ on $C^{m}$. Consequently we can state the following more general theorem:

THeORem 17.2. Let $C$ be a curve of Type W. Let $f^{(p-1)}(z)$ satisfy a Lipschitz condition of order $\alpha-p+1,0 \leqq p-1 \leqq \alpha<p$, on $C$. Then $f^{(p-1)}(z)$ has a bounded derivative of every order $\beta<\alpha$ on $C^{m}$.

In considering the converse we restrict ourselves to analytic Jordan curves.

THEOREM 17.3. Let $C$ be an analytic Jordan curve. Let $f(z)$ have a bounded derivative of order $\alpha>0,0 \leqq p-1 \leqq \alpha<p$, on $C^{m}$. Then $f^{(p-1)}(z)$ satisfies a Lipschitz condition of order $\alpha-p+1$ on $C$.

Since Lipschitz conditions and generalized derivatives are preserved in their orders under conformal transformation of analytic arcs and curves (see $\S 14)$, for $0<\alpha \leqq 1$ the theorem is an immediate consequence of the corresponding result of Weyl [1] for $C$ a segment of the axis of reals. For the general $\alpha$ by Theorem 13.4 we know that $f(z)$ has a bounded derivative of order $\alpha-p+1$, and hence $f^{(p-1)}(z)$ satisfies a Lipschitz condition of order $\alpha-p+1$.

It should be noted here that we must use two different values of $k$ to obtain an overlapping Lipschitz condition. This overlap gives us a Lipschitz condition which is uniform on $C$.

Suppose that $C$ is a Jordan curve composed of a finite number of analytic Jordan arcs meeting in corners of openings $\mu_{k} \pi, k=1,2, \cdots, p, 0<\mu_{k}<2$. By the above result we know that if $f(z)$ has a bounded derivative of order $\alpha>0$ on $C^{m}$, then $f^{(p-1)}(z)$ satisfies a uniform Lipschitz condition of order $\alpha-p+1$ on each analytic arc of $C$. The following lemma serves to connect Lipschitz conditions on two adjacent arcs: 
LEMMA 17.4. Let $C$ be a Jordan arc composed of two analytic arcs meeting in a corner $z_{0}$ of opening $\mu \pi, 0<\mu<2$. Let the function $f(z)$ satisfy a uniform Lipschitz condition of order $\alpha, 0<\alpha \leqq 1$, on each arc separately. Then $f(z)$ satrsfies a uniform Lipschitz condition of order $\alpha$ on the entire arc.

The lemma is an immediate consequence of the convexity of the function $y=x^{\alpha}, x>0$.

\section{Chapter IV}

\section{Generalizations of BernsteIn's AND MarkofF's theorems}

18. Bernstein's and Markoff's theorems. The following theorem is well known:*

THEOREM 18.1. Let $P_{n}(z)$ be a polynomial of degree $n$ in $z$ and let $\left|P_{n}(z)\right| \leqq M$ in the unit circle, $|z| \leqq 1$. Then $\left|P_{n}^{\prime}(z)\right| \leqq M n$ in $|z| \leqq 1$. This bound is attained only by the polynomial $\alpha z^{n},|\alpha|=1$.

Markoff [1] $\dagger$ has proved

THEOREM 18.2. Let $P_{n}(z)$ be a polynomial of degree $n$ in $z$ and let $\left|P_{n}(z)\right| \leqq M$ in the interval $-1 \leqq z \leqq+1$. Then $\left|P_{n}^{\prime}(z)\right| \leqq M n^{2},-1 \leqq z \leqq+1$. This bound is attained only by the polynomial $\alpha \cos n \operatorname{arc} \cos z,|\alpha|=1$.

A result of Bernstein [2, p. 38] on the modulus of the derivative of a polynomial is

THEOREM 18.3. Let $P_{n}(z)$ be a polynomial of degree $n$ in $z$ and let $\left|P_{n}(z)\right| \leqq M$ in the interval $-1 \leqq z \leqq+1$. Then $\left|P_{n}^{\prime}(z)\right| \leqq M n /\left(1-z^{2}\right)^{1 / 2}$.

Montel [1] has extended this to generalized derivatives.

THEOREM 18.4. Let $P_{n}(z)$ be a polynomial of degree $n$ in $z$ and let $\left|P_{n}(z)\right| \leqq M$, $-1 \leqq z \leqq+1$. Then for any point in $\left(-z_{0},+z_{0}\right), 0<z_{0}<1$, we have

$$
\left|P_{n}^{\alpha}(z)\right| \leqq \frac{M(\alpha) n^{\alpha}}{\left(1-z_{0}^{2}\right)^{\alpha / 2}}, \quad \alpha>0, \quad k=+1 .
$$

We are concerned with finding evaluations for $\left|P_{n}^{\alpha}(z)\right|$ on more general point sets. It should be noted that inequality (18.10) is not valid in the entire interval but only on a subset of the given interval. This is characteristic of the results on generalized derivatives. In fact in this chapter our inequalities hold in general only for $|z-k|>m>0$, and in the case of a curve $C$ we shall

* See e.g., Bernstein [2], pp. 44-46.

$\dagger$ Markoff considers only polynomials with real coefficients; Riesz [1] treats the general case. See e.g., Bernstein [2], p. 38. 
denote the set: $z$ on $C,|z-k|>m>0$, by $C^{m}$, as above, and in the case of a region $R$ the set: $z$ in $\bar{R},|z-k|>m>0$, by $\bar{R}^{m}$.

19. Curves of Type $S$. Let $C$ be a curve of Type $S$ in the z-plane and let $\left|P_{n}(z)\right| \leqq M$ on $C$. We shall consider $P_{n}^{\alpha}(z), \alpha>0$, on $C$, where $k$ is an arbitrary point on $C$. By Theorem 15.2 we know that

$$
P_{n}^{\alpha}(z)=\frac{\Gamma(\alpha+1)}{2 \pi i} \int_{\gamma} \frac{P_{n}(t) d t}{(t-z)^{\alpha+1}}, \quad \alpha>0,
$$

where $\gamma$ is a rectifiable Jordan curve passing through $k$ and containing $z$ in its interior. Let $z_{0}$ be an arbitrary point on $C^{m}$ and draw a circle $\delta$ of radius $r<m$ about the point $z_{0}$, the value of $r$ to be determined later. In traversing the curve $C$ in a preassigned direction from $k$ to $z$ let the point $A$ be the first intersection of $\delta$ with $C$. We will choose as the curve $\gamma$ the path from $k$ to $A$, from $A$ around $\delta$ back to $A$, and thence back to $k$ along $C$. It is true that this is not a Jordan curve but reference to the proof of Montel [1] of Theorem 15.1 will make it clear that this path of integration satisfies the conditions of the theorem. Then we have

Thus

$$
\begin{aligned}
P_{n}^{\alpha}\left(z_{0}\right)= & \frac{\Gamma(\alpha+1)}{2 \pi i} \int_{k}^{A} \frac{P_{n}(t) d t}{\left(t-z_{0}\right)^{\alpha+1}}+\frac{\Gamma(\alpha+1)}{2 \pi i} \int_{\delta} \frac{P_{n}(t) d t}{\left(t-z_{0}\right)^{\alpha+1}} \\
& +\frac{\Gamma(\alpha+1) e^{-2 \pi i(\alpha+1)}}{2 \pi i} \int_{k}^{A} \frac{P_{n}(t) d t}{\left(t-z_{0}\right)^{\alpha+1}} .
\end{aligned}
$$

Let

$$
\left|P_{n}^{\alpha}\left(z_{0}\right)\right| \leqq \frac{\Gamma(\alpha+1)}{2 \pi}\left\{2\left|\int_{k}^{A} \frac{P_{n}(t) d t}{\left(t-z_{0}\right)^{\alpha+1}}\right|+\left|\int_{\delta} \frac{P_{n}(t) d t}{\left(t-z_{0}\right)^{\alpha+1}}\right|\right\} .
$$

$$
I^{\prime} \equiv \int_{\delta} \frac{P_{n}(t) d t}{\left(t-z_{0}\right)^{\alpha+1}} \quad \text { and } \quad I^{\prime \prime} \equiv \int_{k}^{A} \frac{P_{n}(t) d t}{\left(t-z_{0}\right)^{\alpha+1}} .
$$

We need a well known result,*

Lemma 19.1. Let $E$ be a closed limited point set whose complement is connected and regular. If the polynomial $P_{n}(z)$ of degree $n$ in $z$ satisfies the inequality $\left|P_{n}(z)\right| \leqq M$ for $z$ on $E$, then we have

$$
\left|P_{n}(z)\right| \leqq M \rho^{n}, \quad \rho>1, \quad z \text { on or within } C_{\rho} .
$$

We know by Theorem 3.1 that $d\left(C, C_{\rho}\right) \geqq M_{1}(\rho-1)$ and hence if we choose $r=M_{1}(\rho-1)$ it follows from the lemma that $\left|P_{n}(z)\right| \leqq M \rho^{n}$ for $z$ on and within the circle $\delta$. Then we have

* For various types of point sets this lemma is due to various authors: see Bernstein [1], Szegö [1], Faber [4], Walsh [1]. We state here the form due to Walsh. 


$$
\left|I^{\prime}\right| \leqq \int_{\delta} \frac{\left|P_{n}(t)\right||d t|}{\left|t-z_{0}\right|^{\alpha+1}} \leqq \frac{M \rho^{n} 2 \pi M_{1}(\rho-1)}{M_{1}^{\alpha+1}(\rho-1)^{\alpha+1}}=\frac{2 \pi M}{M_{1}^{\alpha}} \frac{\rho^{n}}{(\rho-1)^{\alpha}} .
$$

The function $y=\rho^{n} /(\rho-1)^{\alpha}, \rho>1$, has a minimum for $\rho=\rho_{n} \equiv n /(n-\alpha)$. For $n$ sufficiently large $M_{1}\left(\rho_{n}-1\right)=M_{1}[\alpha /(n-\alpha)]$ will be positive and less than $m$, in fact our evaluation will hold for all $n$ satisfying the inequality

$$
M_{1}\left(\frac{\alpha}{n-\alpha}\right)<m, \quad(n-\alpha)>\frac{M_{1} \alpha}{m}, \quad n>\frac{M_{1} \alpha}{m}+\alpha .
$$

Now consider $\rho_{n}{ }^{n} /\left[\left(\rho_{n}-1\right)^{\alpha} n^{\alpha}\right]$. It is easy to see that this function approaches $e^{2 \alpha} \alpha^{-\alpha}$ as $n$ approaches $\infty$ and hence we have

$$
\left|I^{\prime}\right| \leqq M K_{1}(\alpha, C) n^{\alpha} \text {. }
$$

Next

$$
\left|I^{\prime \prime}\right| \leqq M \int_{k}^{A} \frac{|d t|}{\left|t-z_{0}\right|^{\alpha+1}} .
$$

By $\$ 10$ the curve $C$ is of Type $W^{\prime}$, and since $A$ is on the circumference of a circle whose center is $z_{0}$ and whose radius is $M_{1}[\alpha /(n-\alpha)]$, we have

$$
\left|I^{\prime \prime}\right| \leqq M M_{1}\left\{N_{1}\left(\frac{\alpha}{n-\alpha}\right)^{-\alpha}+N_{2}\right\} \leqq M K_{2}(\alpha, C) n^{\alpha},
$$

which is valid for $n$ satisfying the inequality (19.12).

Thus we have established the following theorem:

TheOREM 19.2. Let $C$ be a curve of Type S. Let $P_{n}(z)$ be a polynomial of degree $n$ in $z$ and let $\left|P_{n}(z)\right| \leqq M$ on $C$. Then for $n$ sufficiently large we have

$$
\left|P_{n}^{\alpha}(z)\right| \leqq M K(\alpha, C) n^{\alpha}, \quad \alpha>0, \quad z \text { on } C^{m},
$$

where $K(\alpha, C)$ is a constant depending only on $\alpha$ and $C$.

20. Curves of Type $t$. The method of the preceding paragraph is valid in the case where $C$ is a curve of Type $t$ with only slight modification. In fact the only essential change is the substitution of $\alpha t$ for $\alpha$, since by Theorem 4.3 we have here $d\left(C, C_{\rho}\right) \geqq M_{1}(\rho-1)^{t}$. The result can be stated as follows:

TheORem 20.1. Let $C$ be a curve of Type $t$. Let $P_{n}(z)$ be a polynomial of degree $n$ in $z$ and let $\left|P_{n}(z)\right| \leqq M$ on $C$. Then for $n$ sufficiently large we have (20.10)

$$
\left|P_{n}^{\alpha}(z)\right| \leqq M K(\alpha, C) n^{\alpha t}, \quad \alpha>0, \quad 1 \leqq t<2, \quad z \text { on } C^{m},
$$

where $K(\alpha, C)$ is a constant depending only on $\alpha$ and $C$.

21. Regions of Type $W^{\prime}$. In this case we have $d\left(C, C_{\rho}\right) \geqq M_{1}(\rho-1)^{2}$ by Theorem 6.3, and hence the $t$ in Theorem 20.1 must be replaced by 2 . The 
definition of a region of Type $W^{\prime}(\$ 10)$ then leads directly by an application of the above methods to

THEOREM 21.1. Let $R$ be a region of Type $W^{\prime}$ with boundary $C$. Let $P_{n}(z)$ be a polynomial of degree $n$ in $z$ and let $\left|P_{n}(z)\right| \leqq M$ in $\bar{R}$. Then for $n$ sufficiently large we have

$$
\left|P_{n}^{\alpha}(z)\right| \leqq M K(\alpha, R) n^{2 \alpha}, \quad \alpha>0, \quad z \text { on } C^{m},
$$

where $K(\alpha, R)$ is a consiant depending only on $\alpha$ and $R$.

Here $k$ is a point on $C$ and, as in $\$ \S 19$ and $20, P_{n}^{\alpha}(z)$ is a continuous function of $z$ on $C^{m}$. There is no confusion as to branches since both $k$ and $z$ are boundary points.

22. Another choice of $k$. In the case where $f(z)$ is analytic in a limited simply connected region $R$ with boundary $C$, and continuous in the corresponding closed region, we may choose $k$ not on $C$ but in $R$. Then we can consider all $z$ in $\bar{R}$ such that $|z-k|>m>0$, i.e., in $\bar{R}^{m}$. Of course the value of the derivative depends upon the point $k$ chosen.

The original choice of $k$ on $C$ is more general in that the function need be defined only on the set $C$. However, in the application of these theorems to approximation by polynomials in closed regions the functions are assumed analytic in the regions and continuous in the corresponding closed regions. Under these conditions it is simpler to choose $k$ inside and then we know that the above evaluations hold not only on the boundary but everywhere in the closed region except for the points $z$ such that $|z-k| \leqq m$.

Thus we have the following results:

THEOREM 22.1. Let $R$ be the limited region bounded by a curve $C$ of Type $S$. Let $P_{n}(z)$ be a polynomial of degree $n$ in $z$ and let $\left|P_{n}(z)\right| \leqq M$ in $\bar{R}$. Then for $n$ sufficiently large we have

$$
\left|P_{n}^{\alpha}(z)\right| \leqq M K(\alpha, R) n^{\alpha}, \quad \alpha>0, \quad z \text { in } \bar{R}^{m},
$$

where $K(\alpha, R)$ depends only on $\alpha$ and $R$.

THEOREM 22.2. Let $R$ be the limited region bounded by a curve $C$ of $T y p e t$. Let $P_{n}(z)$ be a polynomial of degree $n$ in $z$ and let $\left|P_{n}(z)\right| \leqq M$ in $\bar{R}$. Then for $n$ sufficiently large we have

$$
\left|P_{n}^{\alpha}(z)\right| \leqq M K(\alpha, R) n^{\alpha t}, \quad \alpha>0, \quad 1 \leqq t<2, \quad z \text { in } \bar{R}^{m},
$$

where $K(\alpha, R)$ depends only on $\alpha$ and $R$.

Theorem 22.3. Let $R$ be a region of Type $W^{\prime}$ with boundary $C$. Let $P_{n}(z)$ be a polynomial of degree $n$ in $z$ and let $\left|P_{n}(z)\right| \leqq M$ in $\bar{R}$. Then for $n$ sufficiently large we have 


$$
\left|P_{n}^{\alpha}(z)\right| \leqq M K(\alpha, R) n^{2 \alpha}, \quad \alpha>0, \quad z \text { in } \bar{R}^{m},
$$

where $K(\alpha, R)$ depends only on $\alpha$ and $R$.

If we choose $k$ in $R$ and $m$ sufficiently small the boundary $C$ will lie in $\bar{R}^{m}$.

23. Ordinary derivatives. If $\alpha$ is a positive integer we have to consider only the part $I^{\prime}$ of the expression for $P_{n}^{\alpha}(z)$ in $\$ 19$. Thus we avoid the question of curves of Type $W^{\prime}$. In this connection Szegö [2] has considered the case where $C$ is a Jordan curve composed of a finite number of analytic arcs. He shows that if $z_{0}$ is a corner whose exterior opening is $\mu \pi, 0<\mu \leqq 2$, and if $\left|P_{n}(z)\right| \leqq M$ on $C$, then $M M_{1} n^{\mu} \leqq\left|P_{n}^{\prime}\left(z_{0}\right)\right| \leqq M M_{2} n^{\mu}$, where $M_{1}$ and $M_{2}$ are constants independent of $n$ (see Theorem 22.2).

Jackson [2] has shown that if the boundary $C$ of a limited simply connected region $R$ is such that through every boundary point $P$ of $C$ a circle of radius $h>0, h$ independent of $P$, can be drawn whose interior lies interior to the region, then $\left|P_{n}(z)\right| \leqq M, z$ in $\bar{R}$, implies $\left|P_{n}^{\prime}(z)\right| \leqq M M_{2} n, z$ in $\bar{R}$, where $M_{2}$ is a constant independent of $M, n$, and $z$; this result follows from Theorem 18.1. Also Jackson [3] has shown that for a limited simply connected region $R$ such that from every boundary point $P$ a straight line of length $h>0$, $h$ independent of $P$, can be drawn which lies entirely in $R$, the inequality $\left|P_{n}(z)\right| \leqq M, z$ in $\bar{R}$, implies $\left|P_{n}^{\prime}(z)\right| \leqq M M_{2} n^{2}, z$ in $\bar{R}$; it is clear that this is a region of Type $W^{\prime}$ and hence the result is a corollary of Theorem 21.1. In this connection the author [3] has shown that if $\left|P_{n}(z)\right| \leqq M$ on a set $E$ with no isolated points and whose complement has finite connectivity, then $\left|P_{n}^{\prime}(z)\right| \leqq M K(E) n^{2}, z$ on $E$, where the constant $K(E)$ depends only on the set $E$.

\section{Chapter V}

\section{Approximation by polynomials-Problem $\alpha$}

24. Analytic Jordan curves. We will prove the following theorem:

THeOREM 24.1. Let $R$ be a limited region bounded by an analytic Jordan curve $C$. Let $f(z)$ be defined in $\bar{R}$. If for every $n$ there exists a polynomial $P_{n}(z)$ of degree $n$ in $z$ such that

$$
\left|f(z)-P_{n}(z)\right|<\frac{M}{n^{\alpha}}, \quad \alpha>0, \quad z \text { in } \bar{R},
$$

$M$ a constant independent of $n$ and $z$, then $f(z)$ is analytic in $R$, continuous in $\bar{R}$, and has a bounded derivative of every order $\alpha^{\prime}<\alpha$ on $C$.

The analyticity and continuity are immediate consequences of (24.10). For the derivative we have to apply our previous results. Hereafter we will choose an interior point of the region as the point $k$ and $m$ so small that every 
point of $C$ belongs to $\bar{R}^{m}$. Using Theorems 22.1 and 16.1 we can apply a procedure identical to that of Montel [1, pp. 175-176] to prove the theorem.

We have in the other direction

TheORem 24.2. Let $R$ be a limited region bounded by an analytic Jordan curve $C$. Let $f(z)$ be analytic in $R$, continuous in $\bar{R}$, and have a bounded derivative of order $\alpha>0$ on $C$. Then there exist polynomials of respective degrees $n$, $n=1,2, \cdots$, such that

$$
\left|f(z)-P_{n}(z)\right| \leqq \frac{M}{n^{\alpha}}, \quad z \text { in } \bar{R},
$$

where $M$ is a constant independent of $n$ and $z$.

Let $0 \leqq p-1 \leqq \alpha<p$, then by Theorem 17.3 we know that $f^{(p-1)}(z)$ satisfies a uniform Lipschitz condition of order $\alpha-p+1$ on $C$. Thus we have only to apply the following theorem of Curtiss [1]:

THEOREM 24.3. Let $E$, with boundary $C$, be a closed limited point set consisting of a finite number of mutually exterior closed Jordan regions, $R_{\mathbf{1}}, R_{\mathbf{2}}, \cdots, R_{s}$, each bounded by an analytic Jordan curve. Let $f(z)$ be a function analytic throughout the interior of $C$, continuous on $E$, and having $a(p-1)$ st derivative which satisfies a Lipschitz condition of order $\alpha-p+1,0<\alpha-p+1 \leqq 1$, on the boundary of $E$. Then there exist polynomials $P_{n}(z)$ of respective degrees $n$, $n=1,2, \cdots$, such that

$$
\left|f(z)-P_{n}(z)\right| \leqq \frac{M}{n^{\alpha}}, \quad z \text { on } E,
$$

where $M$ is a constant independent of $n$ and $z .^{*}$

It should be observed here that Theorem 24.1 taken with Theorem 24.3 leads to a new proof of Theorem 17.1 where $C$ is an analytic Jordan curve.

Also Theorem 17.3 leads to the following result:

THEOREM 24.4. Under the same hypothesis as in Theorem 24.1, let $0 \leqq p-1 \leqq \alpha<p$. Then $f(z)$ is analytic in $R$, continuous in $\bar{R}$, and $f^{(p-1)}(z)$ satisfies a Lipschitz condition of every order $\alpha^{\prime}-p+1, \alpha^{\prime}<\alpha$, on $C$.

25. Curves of Type $S$. In the proof of Theorem 24.1 we apply Theorem 22.1 which does not require $C$ to be analytic but merely of Type $S$ and thus we have the following more general theorem:

Theorem 25.1. Let $R$ be a limited region bounded by a curve $C$ of Type $S$.

* This theorem is a sharpening of a result on degree of convergence of Faber's polynomials established by the author [1]. 
Let $f(z)$ be defined in $\bar{R}$. If for every $n, n=1,2, \cdots$, there exists a polynomial $P_{n}(z)$ of degree $n$ in $z$ such that

$$
\left|f(z)-P_{n}(z)\right| \leqq \frac{M}{n^{\alpha}}, \quad \alpha>0, \quad z \text { in } \bar{R},
$$

$M$ a constant independent of $n$ and $z$, then $f(z)$ is analytic in $R$, continuous in $\bar{R}$, and has a bounded derivative of every order $\alpha^{\prime}<\alpha$ on $C$.

Only in the case where $C$ is analytic has the converse theorem been proved.

26. Curves with corners. Here we consider first curves of Type $t$, and using Theorem 22.2 we can prove precisely as above

THEOREM 26.1. Let $R$ be a limited region bounded by a curve $C$ of $T y p e t$. Let $f(z)$ be defined in $\bar{R}$. If for every $n, n=1,2, \cdots$, there exists a polynomial $P_{n}(z)$ of degree $n$ in $z$ such that

$$
\left|f(z)-P_{n}(z)\right| \leqq \frac{M}{n^{\alpha t}}, \quad \alpha>0, \quad 1 \leqq t<2, \quad z \text {.in } \bar{R},
$$

$M$ a constant independent of $n$ and $z$, then $f(z)$ is analytic in $R$, continuous in $\bar{R}$, and has a bounded derivative of every'order $\alpha^{\prime}<\alpha$ on $C$.

Combining the above result with Theorems 17.3 and 17.4 we can substitute a Lipschitz condition for the generalized derivative.

Theorem 26.2. Let $R$ be a limited region bounded by a Jordan curve $C$ composed of a finite number of analytic arcs meeting in corners of exterior openings $\mu_{k} \pi, 2>\mu_{1} \geqq \mu_{2} \geqq \cdots \geqq \mu_{s}>0$, and let $t=\mu_{1}$ if $\mu_{1} \geqq 1$, and $t=1$ if $\mu_{1}<1$. Let $f(z)$ be defined in $\bar{R}$. If for every $n, n=1,2, \cdots$, there exists a polynomial $P_{n}(z)$ of degree $n$ in $z$ such that

$$
\left|f(z)-P_{n}(z)\right| \leqq \frac{M}{n^{\alpha t}}, \quad 0 \leqq p-1 \leqq \alpha<p, \quad 2>t \geqq 1, \quad z \text { in } \bar{R},
$$

$M$ a constant independent of $n$ and $z$, then $f(z)$ is analytic in $R$, continuous in $\bar{R}$, and $f^{(p-1)}(z)$ satisfies a Lipschitz condition of every order $\alpha^{\prime}-p+1, \alpha^{\prime}<\alpha$, on $C$.

27. Regions of Type $W^{\prime}$. Here the result corresponding to Theorem 26.1 is

THEOREM 27.1. Let $R$ be a region of Type $W^{\prime}$ with boundary $C$ and let $f(z)$ be defined in $\bar{R}$. If for every $n, n=1,2, \cdots$, there exists a polynomial $P_{n}(z)$ of degree $n$ in $z$ such that

$$
\left|f(z)-P_{n}(z)\right| \leqq \frac{M}{n^{2 \alpha}}, \quad \alpha>0, \quad z \text { in } \bar{R},
$$

$M$ a constant independent of $n$ and $z$, then $f(z)$ is analytic in $R$, continuous in $\bar{R}$, and has a bounded derivative of every order $\alpha^{\prime}<\alpha$ on $C$. 
28. Arbitrary closed sets. Theorem 27.1 can be proved for ordinary derivatives on much more general sets than those of \$27. (See Sewell [3].) We do not require the set to be connected; it is sufficient for the set to be closed and limited and have a complement which is connected and regular.

\section{Chapter VI}

\section{APPROXIMATION BY POLYNOMIALS-Problem $\beta$}

29. Uniformly bounded functions. We begin with

THEOREM 29.1. Let $f(z)$ be analytic and uniformly bounded in $|z| \leqq \rho>1$. Then we have

$$
\left|f(z)-T_{n}(z)\right| \leqq \frac{M}{\rho^{n}}, \quad|z| \leqq 1,
$$

where $M$ is a constant independent of $n$ and $z$, and $T_{n}(z)$ is the sum of the first $n$ terms of the Taylor development of $f(z)$ about $z=0$.

We know that

$$
f(z)-T_{n}(z)=\frac{z^{n+1}}{2 \pi i} \int_{|z|=\rho-\epsilon} \frac{f(t) d t}{t^{n+1}(t-z)}, \quad \rho-1>\epsilon>0, \quad|z| \leqq 1 ;
$$

hence

$$
\left|f(z)-T_{n}(z)\right| \leqq \frac{1}{2 \pi} \frac{M_{1} 2 \pi(\rho-\epsilon)}{(\rho-\epsilon)^{n+1}(\rho-\epsilon-1)}=\frac{M_{1}}{(\rho-\epsilon)^{n}(\rho-\epsilon-1)} .
$$

Allowing $\epsilon$ to approach 0 we have (29.10), and the proof is complete.

The following theorem is an extension of the above result to analytic Jordan curves:

THEOREM 29.2. Let $C$ be an analytic Jordan curve bounding the limited region $R$. Let $f(z)$ be analytic and uniformly bounded, $|f(z)| \leqq M$, interior to $C_{\rho}, \rho>1$. Then we have

$$
\left|f(z)-\sum_{0}^{n} a_{\nu} P_{\nu}(z)\right| \leqq \frac{A M}{\rho^{n}}, \quad z \text { in } \bar{R},
$$

where $A$ is a constant depending only on $\rho$ and $C$, and $P_{\nu}(z)$ is the Faber* polynomial of degree $\nu$ belonging to $R$.

Let $z=\psi(w)$ map the exterior of $C$ on the exterior of $\gamma:|w|=1$, so that the

* See Faber $[1,2,3]$. It should be pointed out that there is a slight error in the recurrence formula which is given for $P_{\boldsymbol{v}}(z)$ in Faber [3]; formula (15) should contain an additional constant due to the fact that the power series $\mathfrak{B}_{n}(t)$ in formula (13) may begin with a non-zero term in $t$ to the 0 power. 
points at $\infty$ correspond to each other. Then from the results of Faber [3] we know that $f(z)=\sum_{0}^{\infty} a_{\nu} P_{\nu}(z), z$ interior to $C_{\rho}$, where

$$
P_{\nu}(z)=c^{\nu} w^{\nu}\left(1+\theta_{\nu}(z) G\right),
$$

$G$ a fixed constant, $c$ the capacity* of $R$, and $\left|\theta_{\nu}(z)\right|<1$ for $z$ interior to $C_{\rho}$. Also by Faber [3],

$$
\left|a_{\nu}\right|=\left|\frac{1}{2 \pi i} \int_{|w|=\rho-\epsilon} \frac{f[\psi(\omega)] d \omega}{c^{\nu} \omega^{\nu+1}}\right| \leqq \frac{1}{2 \pi} \frac{M 2 \pi(\rho-\epsilon)}{c^{\nu}(\rho-\epsilon)^{\nu+1}}=\frac{M}{c^{\nu}(\rho-\epsilon)^{\nu}},
$$

and letting $\epsilon$ approach 0 we have

$$
\left|a_{\nu}\right| \leqq \frac{M}{c^{\nu} \rho^{\nu}}
$$

Furthermore from (29.12) we have

$$
\left|P_{\nu}(z)\right| \leqq c^{\nu}\left[1+\theta_{\nu}(z) G\right], \quad z \text { on } C .
$$

Hence

$$
\begin{aligned}
\left|f(z)-\sum_{0}^{n} a_{\nu} P_{\nu}(z)\right| & =\left|\sum_{n+1}^{\infty} a_{\nu} P_{\nu}(z)\right| \leqq \sum_{n+1}^{\infty} \frac{M\left[1+\theta_{\nu}(z) G\right]}{\rho^{\nu}} \\
& \leqq M M^{\prime} \sum_{n+1}^{\infty} \frac{1}{\rho^{\nu}} \leqq \frac{A M}{\rho^{n}}, \quad z \text { on } C,
\end{aligned}
$$

and the proof is complete.

30. Lipschitz conditions, generalized derivatives, and degree of approximation. We start here with the unit circle and prove the following theorem:

THeOREM 30.1. Let $f(z)$ be analytic in $|z|<\rho>1$, continuous in $|z| \leqq \rho$, and have a bounded derivative of order $\alpha>0$ on $C_{\rho},|z|=\rho$. Then we have

$$
\left|f(z)-T_{n}(z)\right| \leqq \frac{M \log n}{n^{\alpha} \rho^{n}}, \quad|z| \leqq 1,
$$

where $T_{n}(z)$ is the sum of the first $n$ terms of the Taylor development of $f(z)$ about $z=0$.

CASE I. $0<\alpha \leqq 1$. Since $f(z)$ has a bounded derivative of order $\alpha$ it satisfies a Lipschitz condition of order $\alpha$ by Theorem 17.3. Make the transformation $z=\rho y$, then $f(z) \equiv f(\rho y) \equiv \Phi(y)$, and $\Phi(y)$ satisfies a Lipschitz condition of order $\alpha$ on $|y|=1$. We need the following result due to the author [1]:

* See $§ 7$. Faber maps the exterior of $C$ on the interior of the circle whose radius is the reciprocal of $c$. The relation (7.11) enters in this connection. 
LEMma 30.2. If $f(z)$ is analytic in $|z|<1$, continuous in $|z| \leqq 1$, and if $f^{(p-1)}(z)$ satisfies a Lipschitz condition of order $\alpha-p+1,0<\alpha-p+1 \leqq 1$, on $|z|=1$, then we have

$$
\left|f(z)-T_{n}(z)\right| \leqq \frac{M_{1} \log n}{n^{\alpha}}, \quad|z| \leqq 1,
$$

where $T_{n}(z)$ is the sum of the first $n$ terms of the Taylor development of $f(z)$ about $z=0$.

By the lemma

$$
\left|\Phi(y)-t_{n}(y)\right| \leqq \frac{M_{1} \log n}{n^{\alpha}}, \quad|y| \leqq 1,
$$

where $t_{n}(y)$ is the sum of the first $n$ terms of the Taylor development of $\Phi(y)$ about $y=0$. Since $\Phi(y)-t_{n}(y)$ has a zero of multiplicity at least as great as $n+1$ at $y=0$, we have by Schwarz' lemma*

$$
\left|\Phi(y)-t_{n}(y)\right| \leqq \frac{M_{1} \log n}{n^{\alpha}}|y|^{n+1}, \quad|y| \leqq 1,
$$

and transforming back to the $z$-plane we have

$$
\left|f(z)-T_{n}(z)\right| \leqq \frac{M_{1} \log n}{n^{\alpha} \rho^{n+1}}=\frac{M \log . n}{n^{\alpha} \rho^{n}}, \quad|z| \leqq 1 .
$$

CASE II. $0 \leqq p-1 \leqq \alpha<p$. If $f(z)$ has a bounded derivative of order $\alpha$ on $C_{\rho}$, then by Theorem 17.3 the function $f^{(p-1)}(z)$ satisfies a Lipschitz condition of order $\alpha-p+1$ on $C_{\rho}$. Thus the same method as above yields

$$
\left|f(z)-T_{n}(z)\right| \leqq \frac{M \log n}{n^{\alpha-p+1+p-1} \rho^{n}}=\frac{M \log n}{n^{\alpha} \rho^{n}}, \quad|z| \leqq 1,
$$

and the proof is complete.

As an immediate consequence of a theorem of the author [1] and Theorem 17.3 we have

THEOREM 30.3. Let $C$ be an analytic Jordan curve and let $f(z)$ be analytic interior to $C$ and continuous in the corresponding closed region bounded by $C$, and have a bounded derivative of order $\alpha>0$ on $C$. Then we have

$$
\left|f(z)-\sum_{0}^{n} a_{\nu} P_{\nu}(z)\right| \leqq \frac{M \log n}{n^{\alpha}}, \quad z \text { on } C,
$$

where $P_{\nu}(z)$ is the Faber polynomial of degree $\nu$ belonging to $C$. ably.

* I am indebted to the referee for this suggestion, which shortens my original proof consider- 
An application of this theorem leads to

Theorem 30.4. Let $C$ be an analytic Jordan curve and let $f(z)$ be analytic interior to $C_{\rho}, \rho>1$, continuous in the corresponding closed region, and have a bounded derivative of order $\alpha>0$ on $C_{\rho}$. Then we have

$$
\left|f(z)-\sum_{0}^{n} a_{\nu} P_{\nu}(z)\right| \leqq \frac{M \log n}{n^{\alpha} \rho^{n}}, \quad z \text { on } C,
$$

where $P_{\nu}(z)$ is the Faber polynomial of degree $\nu$ belonging to $C$.

Since the Faber development is unique the Faber development of the function

$$
\Phi(z)=f(z)-\sum_{.0}^{n} a_{\nu} P_{\nu}(z)
$$

is $\sum_{n+1}^{\infty} a_{\nu} P_{\nu}(z)$. Applying Theorem 29.2 to the function $\Phi(z)$ which on $C_{\rho}$ is in modulus less than $M \log n / n^{\alpha}$ (by Theorem 30.2), we immediately obtain the desired result.*

31. The converse problem. In all of the theorems of $\$ 30$ we assume conditions on the function $f(z)$ on $C_{\rho}$ and find the degree of approximation on $C$. In the following theorem we consider the converse problem:

Theorem 31.1. Let $E$, with boundary $C$, be a closed limited point set whose complement is simply connected. Let $f(z)$ be analytic on $E$. If there exists a polynomial $P_{n}(z)$ of degree $n, n=1,2, \cdots$, such that

$$
\left|f(z)-P_{n}(z)\right| \leqq \frac{M}{n^{\alpha+1} \rho^{n}}, \quad \alpha>0, \quad \rho>1, \quad z \text { on } E,
$$

where $M$ is a constant independent of $n$ and $z$, then $f(z)$ is analytic interior to $C_{\rho}$, continuous in the closed limited region bounded by $C_{\rho}$, and has a bounded derivative of every order $\alpha^{\prime}<\alpha$ on $C_{\rho}$.

The analyticity of $f(z)$ interior to $C_{\rho}$ and its continuity in $\bar{C}_{\rho}$ are immediate consequences of (31.10). For the bounded derivative we know by hypothesis that

$$
\begin{aligned}
& \left|f(z)-P_{n}(z)\right| \leqq \frac{M}{n^{\alpha+1} \rho^{n}}, \quad z \text { on } E \\
& \left|f(z)-P_{n+1}(z)\right| \leqq \frac{M}{(n+1)^{\alpha+1} \rho^{n+1}}, \quad z \text { on } E
\end{aligned}
$$

* This proof of the theorem was suggested by the referee. 
hence

$$
\left|P_{n+1}(z)-P_{n}(z)\right| \leqq \frac{2 M}{n^{\alpha+1} \rho^{n}}, \quad z \text { on } E,
$$

and consequently by Lemma 19.1 we have

$$
\left|P_{n+1}(z)-P_{n}(z)\right| \leqq \frac{2 M \rho^{n+1}}{n^{\alpha+1} \rho^{n}}, \quad z \text { on } C_{\rho} .
$$

Since $C_{\rho}$ is an analytic Jordan curve, we can apply Theorem 22.1 to obtain the inequality

$$
\begin{aligned}
\left|P_{n+1}^{\alpha^{\prime}}(z)-P_{n}^{\alpha^{\prime}}(z)\right| & \leqq \frac{2 M K\left(\alpha^{\prime}, C_{\rho}\right) \rho(n+1)^{\alpha^{\prime}}}{n^{\alpha+1}} \\
& =\frac{M_{1}}{n^{\alpha-\alpha^{\prime}+1}} .
\end{aligned}
$$

By hypothesis $\alpha-\alpha^{\prime}>0$ and we have uniform convergence, and an application of Theorem 16.1 completes the proof.

In this theorem we assume in the denominator an $n$ to the power $\alpha+1$, whereas in Theorem 30.4 we obtain in the denominator only an $n$ to the power $\alpha$. This seems to indicate that the hypothesis in Theorem 31.1 is stronger than necessary, but the following example, suggested by Walsh, shows that the exponent $\alpha+1$ cannot be replaced by a smaller exponent:

Let $E$ be the set $|z| \leqq 1$, and $C_{\rho}$ the circle $|z|=\rho>1$, and consider the function $f(z)=1 /(\rho-z)$. We know that

$$
f(z)-p_{n}(z)=\frac{1}{\rho}\left[\frac{z^{n+1}}{\rho^{n+1}}+\frac{z^{n+2}}{\rho^{n+2}}+\cdots\right], \quad z \text { on } E,
$$

where $p_{n}(z)$ is a polynomial of degree $n$ in $z$. Integrating both sides of (31.11) from 0 to $z$, we have

$$
F(z)-P_{n}(z)=\frac{1}{n \rho^{n}}\left[\frac{n z^{n+2}}{(n+2) \rho}+\frac{n z^{n+3}}{(n+3) \rho^{2}}+\cdots\right], \quad z \text { on } E .
$$

The series in brackets converges and consequently

$$
\left|F(z)-P_{n}(z)\right| \leqq \frac{M}{n \rho^{n}}, \quad z \text { on } E,
$$

and yet the function $F(z)=\log \rho-\log (\rho-z)$ has a logarithmic singularity on $C_{\rho}$ and its derivative has a pole of the first order on $C_{\rho}$. Integrating again we obtain 


$$
\left|F^{*}(z)-P_{n}^{*}(z)\right| \leqq \frac{M}{n^{2} \rho^{n}}, \quad z \text { on } E \text {. }
$$

The first derivative of $F^{*}(z)$ has a logarithmic singularity at $z=\rho$ and hence the function does not have a bounded derivative of order 1 on $C_{\rho}$; also we can show that $F(z)$ does not have a bounded derivative of order $\alpha, 0<\alpha<1$, on $C_{\rho}$, and that $F^{*}(z)$ does not have a bounded derivative of any order $\alpha \geqq 1$ on $C_{\rho}$. On the other hand a direct evaluation shows that $F^{*}(z)$ has a bounded derivative of any order less than 1 on $C_{\rho}$.

This example also shows that the existence of polynomials converging to a function on $C$ with an error less than $M /\left(n \rho^{n}\right)$ does not imply even the continuity of the function on $C_{\rho}$; here $F(z)$ has a logarithmic singularity on $C_{\rho}$. We see further that we have an error less than $M / \rho^{n}$ on $C$ for a function with a single pole of the first order on $C_{\rho}$; in this connection the following result on best approximation is of interest:

Let $C$ be an analytic Jordan curve and let $f(z)$ be analytic on and interior to $C_{\rho}$ except for a finite number of poles on $C_{\rho}$ as follows: $z_{1}, z_{2}, \cdots, z_{s}$, of orders $h_{1}, h_{2}, \cdots, h_{s}$, respectively, where $h_{1} \geqq h_{2} \geqq \cdots \geqq h_{s}>0$. Let $K_{n}(z)$ be that polynomial of degree at most $n$ for which

$$
\epsilon_{n}=\max _{z \text { on } C}\left|f(z)-K_{n} \dot{(z)}\right|
$$

is as small as possible. Then

$$
\frac{n^{h_{1}-1} B_{1}}{\rho^{n}} \leqq \epsilon_{n} \leqq \frac{n^{h_{1}-1} B_{2}}{\rho^{n}},
$$

where $B_{1}$ and $B_{2}$ are constants independent of $n$ and $z$, so long as $z$ is on $C$. Faber [3, pp. 105-106] establishes this result in the case of a single pole of the first order on $C_{\rho}$ and since the method is entirely applicable to the general case the details will not be included here. $\dagger$

AhlFors, L. V.

\section{BIBLIOGRAPHY}

1. Untersuchungen zur Theorie der konformen Abbildung und der ganzen Funktionen, Acta Societatis Scientiarum Fennicae, Nova Series A, vol. 1, (1930), pp. 5-40. Bernstein, $\mathrm{S}$.

1. Sur l'ordre de la meilleure approximation des fonctions continues par des polynomes de degré donné, Mémoire de l'Académie Royale de Belgique, (2), vol. 4, pp. 1-104.

2. Leşons sur les propriétés extrémales et la meilleure approximation des fonctions analytiques d'une variable réele, Paris, 1926.

Carathéodory, C.

1. Conformal Representation, Cambridge Tracts in Mathematics and Mathematical Physics, No. 28, 1932.

$\dagger$ For $E$ a segment of the axis of reals see Bernstein [2], Chap. 3. 
CuRTISs, JoHN.

1. A note on the degree of polynomial approximation, Bulletin of the American Mathematical Society, vol. 42 (1936), pp. 29-30, Abstract No. 29.

Doetsch, G.

1. Probleme aus der Theorie der Wärmteilung, $V$ Mitteilung, Explizite Lösung des BolometerprobFABER, G. lems, Mathematische Zeitschrift, vol. 28 (1928), pp. 567-578.

1. Über polynomische Entwickelungen, I, Mathematische Annalen, vol. 57 (1903), pp. 389-408.

2. Über polynomische Entwickelungen, II, ibid., vol. 64 (1907), pp. 118-135.

3. Über Tchebyscheffsche Polynome, Journal für die reine und angewandte Mathematik (Crelle), vol. 150 (1920), pp. 79-106.

4. Über nach Polynomen fortschreitenden Reihen, Sitzungsberichte der mathematisch-physikalischen Klasse der Bayerischen Akademie der Wissenschaften zu München, 1922, pp. 157178.

HARDY, G. H., AND LITTLEWOOD, J. E.

1. Some properties of fractional integrals, I, Mathematische Zeitschrift, vol. 27 (1928), pp. 565606.

2. Some properties of fractional integrals, II, ibid., vol. 34 (1932), pp. 403-439.

JACKSON, DUNHAM.

1. The Theory of Approximation, American Mathematical Society Colloquium Publications, vol. 11, 1930.

2. On certain problems of approximation in the complex domain, Bulletin of the American Mathematical Society, vol. 36 (1930), pp. 851-857.

3. On the application of Markoff's theorem to problems of approximation in the complex domain, ibid., vol. 37 (1931), pp. 883-890.

KeLlogG, O. D.

1. Harmonic functions and Green's integral, these Transactions, vol. 13 (1912), pp. 109-132. LEVY, P.

1. Sur la dérivation et l'intégration généralisée, Bulletin des Sciences Mathématiques de France, vol. 58 (1923), pp. 307-320.

LINDELÖF, E.

1. Sur un principe général de l'analyse, Acta Societatis Scientiarum Fennicae, vol. 46, no. 4 (1915), pp. 1-35.

Liouville, J.

1. Sur quelques questions de géométrie et de mécanique, et sur un nouveau genre de calcul pour résoudre ces questions, Journal de l'École Polytechnique, sér. 1, vol. 13, no. 21 (1832), pp. $1-69$.

MaRKoff, A.

1. Sur une question posée par Mendelieff; Bulletin of the Academy of Sciences of Saint PetersMonTeL, $\mathrm{P}$. burg, vol. 62 (1889), pp. 1-24.

1. Sur les polynomes d'approximation, Bulletin de la Société Mathématique de France, vol. 46 (1919), pp. 151-196.

OsGood, W. F., AND TAYLOR, E. H.

1. Conformal transformations on the boundaries of their regions of definition, these Transactions, RIEMANN, B. vol. 14 (1913), pp. 277-298.

1. Gesammelte Mathematische Werke und Wissenschaftlicher Nachlass, Leipzig, 1892, pp. 331-344. Riesz, $M$.

1. Eine trigonometrische Interpolationsformel und einige Ungleichungen für Polynome, Jahresbericht der Deutscher Mathematiker Vereinigung, vol. 23 (1914), pp. 354-368.

2. Über einen Satz des Herrn Serge Bernstein, Acta Mathematica, vol. 40 (1916), pp. 337-347. 
SEIDEL, W.

1. Über die Ränderzuordnung bei konformen Abbildungen, Mathematische Annalen, vol. 104 (1931), pp. 182-243.

SEWELL, W. E.

1. Degree of approximation by polynomials to continuous functions, Bulletin of the American Mathematical Society, vol. 41 (1935), pp. 111-117.

2. Generalized derivatives and approximation, Proceedings of the National Academy of Sciences, vol. 21 (1935), pp. 255-258.

3. On the modulus of the derivative of a polynomial, Bulletin of the American Mathematical SoSzEGö, G. ciety, vol. 42 (1936), pp. 699-701.

1. Über trigonometrische und harmonische Polynome, Mathematische Annalen, vol. 79 (1918), pp. 323-339.

2. Über einen Satz von A. Markoff, Mathematische Zeitschrift, vol. 23 (1925), pp. 45-61.

3. Zur Theorie der schlichten Abbildungen, Mathematische Annalen, vol. 100 (1928), pp. 188-211. TAMARKIN, J. D.

1. On integrable solutions of Abel's integral equation, Annals of Mathematics, (2), vol. 31 (1930), VISSER, C. pp. 219-229.

1. Über beschränkte analytische Funktionen und die Randverhältnisse bei konformen Abbildungen, Mathematische Annalen, vol. 105 (1932), pp. 28-39.

WALSH, J. L.

1. Interpolation and Approximation by Rational Functions in the Complex Domain, American Mathematical Society Colloquium Publications, vol. 20, 1935.

Walsh, J. L., AND SEwell, W. E.

1. Sufficient conditions for various degrees of convergence by polynomials, Bulletin of the American Mathematical Society, vol. 42 (1936), p. 342, Abstract No. 236.

WARSCHAWSKI, $\mathrm{S}$.

1. Über einen Satz von O. D. Kellogg, Nachrichten von der Gesellschaft der Wissenschaften zu Göttingen, Mathematisch-Physikalische Klasse, 1932, pp. 73-86.

2. Über die Randverhalten der Ableitung der Abbildungsfunktion bei konformer Abbildung, MatheWEYL, H. matische Zeitschrift, vol. 35 (1932), pp. 321-456.

1. Bemerkungen zum Begriff des Differentialquotienten gebrochener Ordnung, Vierteljahrschrift der Naturforschenden Gesellschaft in Zürich, vol. 62 (1917), pp. 296-302.

HARVARD UNIVERSITY,

Cambridge, Mass. 